\title{
Aberrant TGF- $\beta$ activation in bone tendon insertion induces enthesopathy-like disease
}

\author{
Xiao Wang, ${ }^{1}$ Liang Xie, ${ }^{1,2}$ Janet Crane, ${ }^{1}$ Gehua Zhen, ${ }^{1}$ Fengfeng Li, ${ }^{1}$ Ping Yang, ${ }^{1,3}$ Manman Gao, ${ }^{1,4}$ Ruoxian Deng, ${ }^{1}$ Yiguo Wang, ${ }^{1}$ \\ Xiaohua Jia, ${ }^{1,5}$ Cunyi Fan, ${ }^{6}$ Mei Wan, ${ }^{1}$ and Xu Cao ${ }^{1}$ \\ 'Department of Orthopedic Surgery, School of Medicine, Johns Hopkins University, Baltimore, Maryland, USA. 25tate Key Laboratory of Oral Diseases, West China Hospital of Stomatology, Sichuan \\ University, Chengdu, Sichuan, China. ${ }^{3}$ Department of Obstetrics and Gynecology, First Affiliated Hospital, School of Medicine, Shihezi University, Shihezi, Xinjiang, China. ${ }^{4}$ Department of Spinal Surgery/ \\ Orthopedic Research Institute, First Affiliated Hospital, Sun Yat-sen University, Guandong, China. ${ }^{5}$ Key Laboratory of Molecular Imaging, Institute of Automation, Chinese Academy of Sciences, Beijing, China. \\ ${ }^{6}$ Department of Orthopedic Surgery, Shanghai Sixth People's Hospital, Shanghai, China.
}

\begin{abstract}
Enthesopathy is a disorder of bone, tendon, or ligament insertion. It represents one-fourth of all tendon-ligament diseases and is one of the most difficult tendon-ligament disorders to treat. Despite its high prevalence, the exact pathogenesis of this condition remains unknown. Here, we show that TCF- $\beta$ was activated in both a semi-Achilles tendon transection (SMTS) mouse model and in a dorsiflexion immobilization (DI) mouse model of enthesopathy. High concentrations of active TCF- $\beta$ recruited mesenchymal stromal stem cells (MSCs) and led to excessive vessel formation, bone deterioration, and fibrocartilage calcification. Transgenic expression of active TCF- $\beta 1$ in bone also induced enthesopathy with a phenotype similar to that observed in SMTS and DI mice. Systemic inhibition of TCF- $\beta$ activity by injection of 1D11, a TCF- $\beta$-neutralizing antibody, but not a vehicle antibody, attenuated the excessive vessel formation and restored uncoupled bone remodeling in SMTS mice. 1D11-treated SMTS fibrocartilage had increased proteoglycan and decreased collagen $X$ and matrix metalloproteinase 13 expression relative to control antibody treatment. Notably, inducible knockout of the TCF- $\beta$ type II receptor in mouse MSCs preserved the bone microarchitecture and fibrocartilage composition after SMTS relative to the WT littermate controls. Thus, elevated levels of active TCF- $\beta$ in the enthesis bone marrow induce the initial pathological changes of enthesopathy, indicating that TGF- $\beta$ inhibition could be a potential therapeutic strategy.
\end{abstract}

\section{Introduction}

The connection of muscles/bones to bones via tendons or ligaments is vital for movement. The entheses, the insertion sites of tendons or ligaments to bones, are essential for a smooth mechanical transfer of load (1). Disorders involving the attachment of connective tissue to bone are referred to as enthesopathy, resulting in a disturbance of the load transfer (2). Over half of sports injuries involve tendons and entheses $(3,4)$. Tendon disorders, which include enthesopathy, represent a considerable socioeconomic burden, estimated to be $\$ 850$ billion per year in health care costs and indirect lost wage expenditures according to the American Public Health Association $(5,6)$. Enthesopathy not only results from injuries including those from sports, but can be an important feature of a considerable number of other disorders and pathogeneses, such as spondyloarthropathy. There are 2 types of entheses (7), fibrous entheses and fibrocartilaginous entheses. Fibrous entheses, such as medial collateral ligament enthesis in the knee, provide a direct attachment of the tendon or ligament to the bone via the periosteum. Fibrocartilaginous entheses, such as Achilles tendon enthesis and anterior cruciate ligament enthesis, are the more predominant type of entheses in humans, composed of 4 transitional zones including tendon/ligament (com-

Conflict of interest: The authors have declared that no conflict of interest exists. Submitted: July 10, 2017; Accepted: December 7, 2017.

Reference information: J Clin Invest. 2018;128(2):846-860.

https://doi.org/10.1172/JCI96186. prising parallel collagen fibers and sparse spindle-like fibroblasts), uncalcified fibrocartilage (UF) (with cell morphology changing to rounded chondrocytes), calcified fibrocartilage (CF) (with characteristic calcified extracellular matrix $[\mathrm{ECM}]$ and separated from UF by a tidemark), and bone (with highly organized lamellar structure and separated from the adjacent CF via a cement line) with graded mechanical properties (8-10).

Enthesopathy can be defined histologically by features, including fibrocartilage degeneration, microfracture, and/or bone necrosis, angiogenesis, and disruption of the tidemark (11-13). In healthy fibrocartilage, type II collagen is the most abundant collagenous ECM protein along with proteoglycans (14-18). ECM turnover remains at relatively low rates and resists proliferation and differentiation. During degeneration, type X collagen (COLX) and MMP13 are overexpressed in fibrocartilage with lower amounts of proteoglycans, decreased UF zone, and expanded CF zone (19-22). Blood vessels and bony defects are also often present, and the blood vessels may pass between the tendon/ligament and the bone marrow (23). Abnormal bony projections at the attachment of a tendon or ligament, known as enthesophytes, may occur at the late stage and can be observed radiographically. Calcaneal enthesophytes at the insertion of the plantar aponeurosis are seen in $25 \%$ of the general population (24-26).

Despite the well-described histological features of enthesopathy, little is known about the cell-signaling mechanisms that contribute to its pathogenesis. The role of TGF- $\beta$ in musculoskeletal 
A SMTS
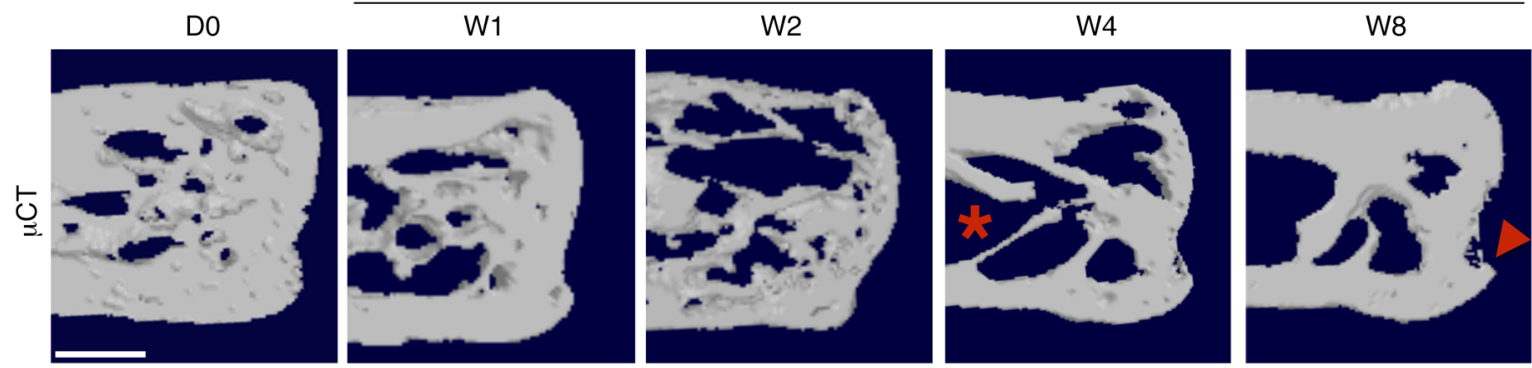

B
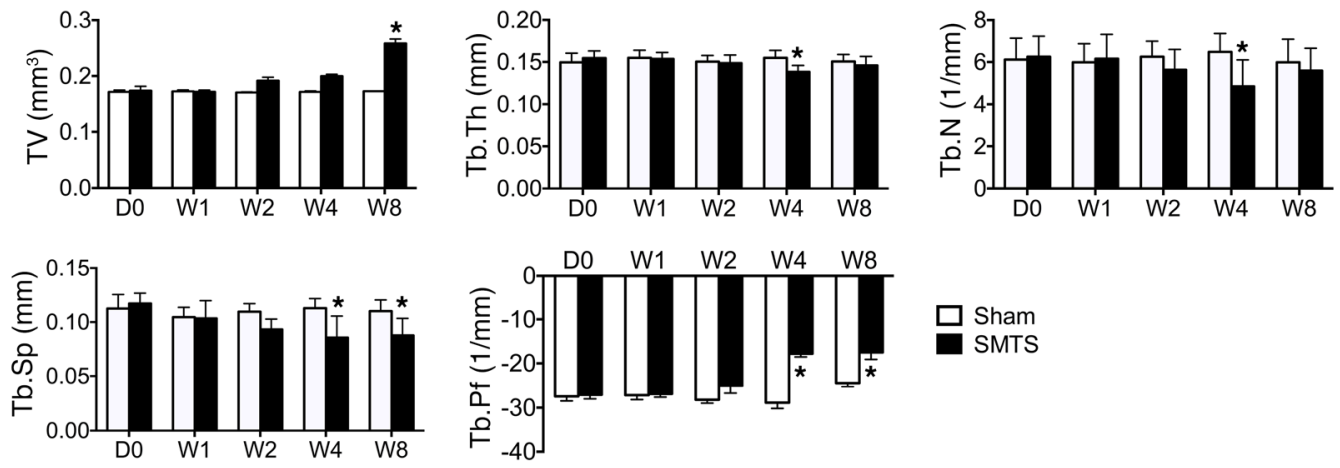

C

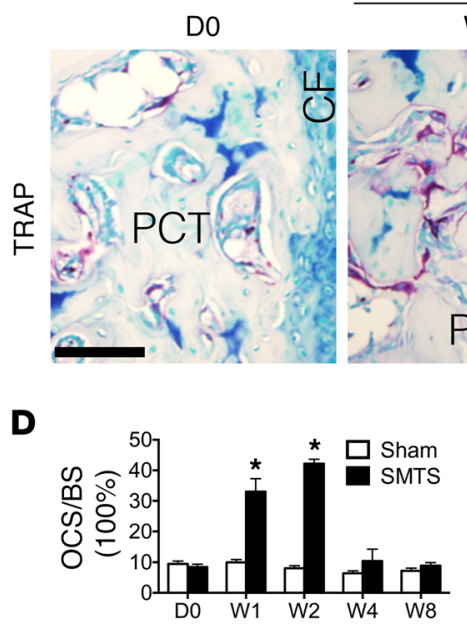

SMTS
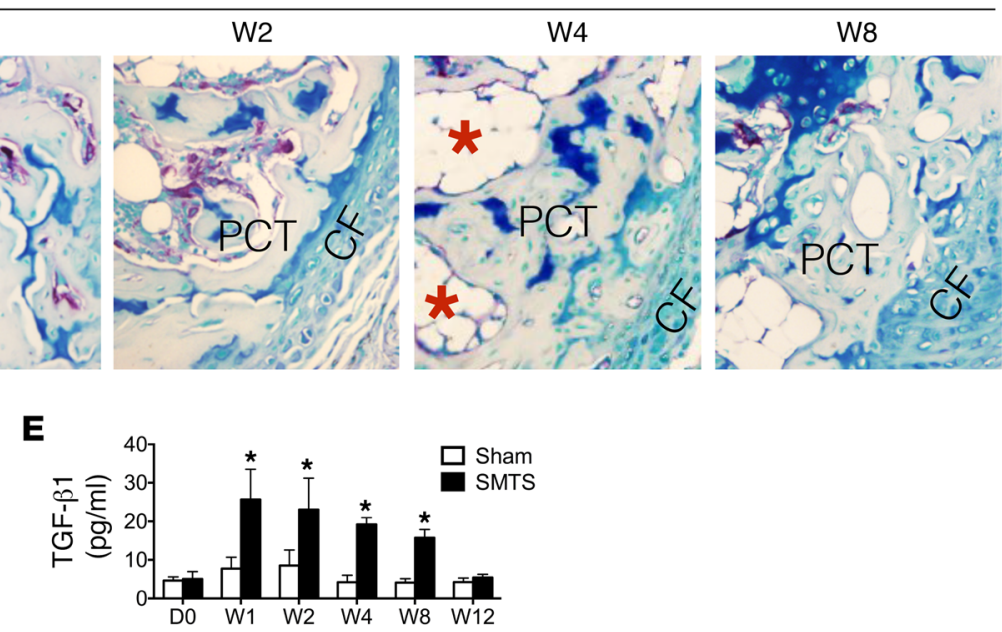

Figure 1. Osteoclastic bone resorption in SMTS enthesopathy mice. (A) $\mu C T$ images of the PCT (sagittal view). Red arrowhead indicates altered morphology of the PCT. Red asterisk indicates bone marrow cavities. Scale bar: $500 \mu \mathrm{m}$. (B) Quantitative analysis of BV/TV, Tb.Th, Tb.N, Tb.Sp, and Tb.Pf in PCT determined by $\mu$ CT analysis. (C) TRAP staining (magenta) in mouse PCT bone marrow. Scale bar: $200 \mu \mathrm{m}$. Red asterisks indicate bone marrow cavities. (D) Quantitative analysis of TRAP+ OCS/BS. (E) Quantitative analysis of active TCF- $\beta 1$ in serum by ELISA. D0, prior to SMTS surgery; Sham, sham surgery; W1, 1 week after SMTS surgery. Data shown as mean \pm SEM. $n=10 .{ }^{*} P<0.05$ compared with the sham group.

disease has drawn increasing attention in recent years. During tissue injury or remodeling, TGF- $\beta$ is activated to recruit stem/ progenitor cells to maintain tissue homeostasis (27-31). We have previously shown that TGF- $\beta$ is released from the bone matrix and activated during osteoclastic bone resorption (31). Active TGF- $\beta$ induces mesenchymal stromal stem cell (MSC) migration to the bone-resorptive pits for new bone formation (31). However, excessive active TGF- $\beta$ leads to uncoupled bone remodeling, contributing to the pathogenesis of rare genetic skeletal diseases such as Camurati-Engelmann disease (CED), osteogenesis imperfecta, and more common musculoskeletal disorders, such as osteoarthritis (31-36). High levels of TGF- $\beta$ have been observed locally and systemically in spondyloarthropathy patients with enthesopathy $(37,38)$. In this study, we determined the role of TGF- $\beta$ in Achilles tendon enthesopathy using 3 different mouse models. We found that the changes of calcaneal bone microstructure and composition were associated with the onset of Achilles tendon enthesopathy. Inhibition of TGF- $\beta$ activity, using either a TGF- $\beta$-neutralizing antibody (1D11) or a conditional knockout mouse model, attenuated calcaneal and fibrocartilage pathological changes.

\section{Results}

Upregulated TGF- $\beta$ activities in posterior calcaneal tuberosity in Achillestendon enthesopathymouse models. To examine the changes at the 
A

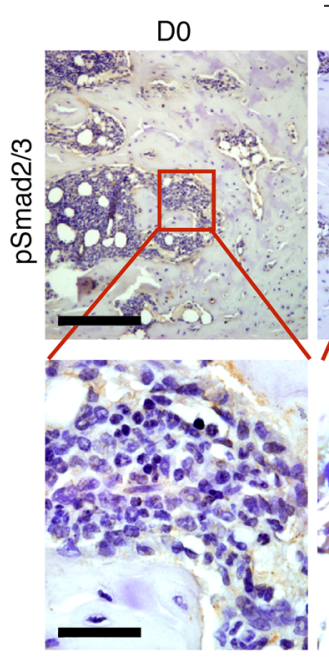

SMTS

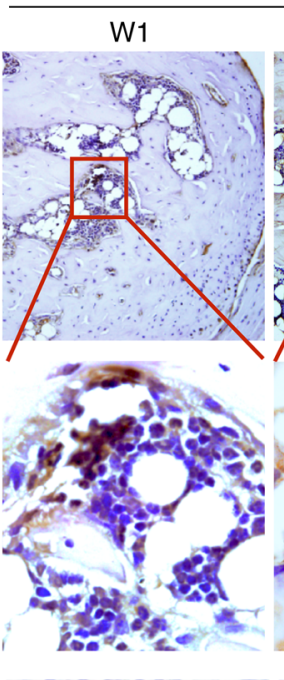

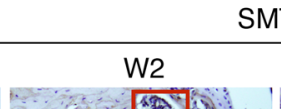

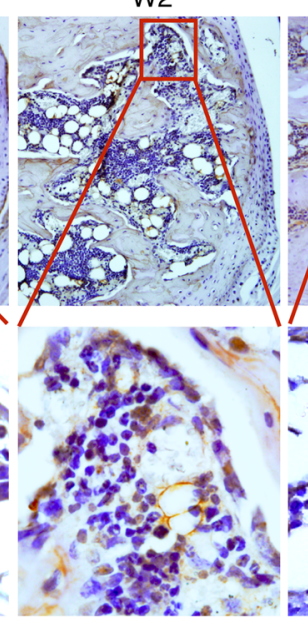

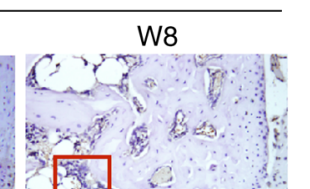

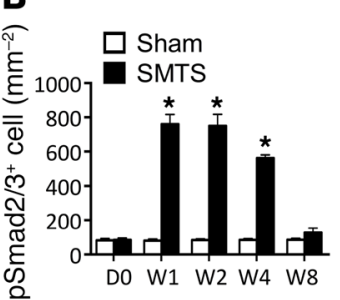

D0 W1 W2 W4 W8
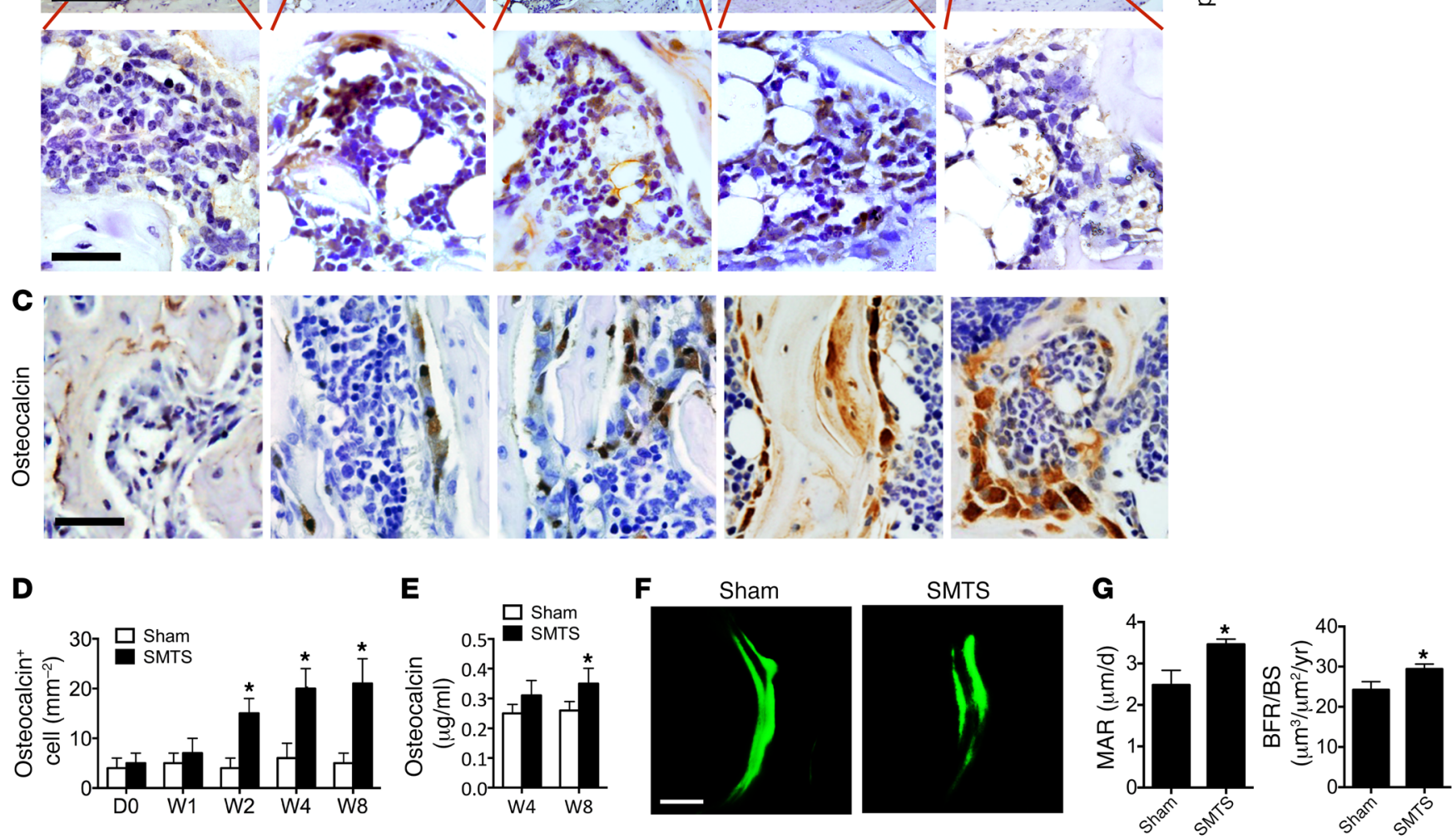

Figure 2. Dysregulated TGF- $\beta$ signaling is associated with trabecular bone changes in SMTS enthesopathy mice. (A) Immunohistochemical analysis of $\mathrm{pSmad} 2 / 3^{+}$cells (brown) in mouse PCT bone marrow. The bottom panels show higher magnification of the boxed area in the top panels. Scale bar: $500 \mu \mathrm{m}$ (top panels); $50 \mu \mathrm{m}$ (bottom panels). (B) Quantitative analysis of the number of pSmad2/3+ cells per bone marrow area (mm²). (C) Immunostaining and (D) quantitative analysis of $\mathrm{Ocn}^{+}$cells. Scale bar: $50 \mu \mathrm{m}$. (E) Quantitative analysis of osteocalcin in serum by ELISA. (F) Representative images of calcein double labeling of Achilles tendon enthesis 8 weeks after sham or SMTS operation with (G) quantification of MAR and BFR. Scale bar: $50 \mu \mathrm{m}$. Data shown as mean \pm SEM. $n=10$. ${ }^{*} P<0.05$ compared with the sham group.

onset of enthesopathy, we generated these 2 models based on prior theories that enthesopathy results from unbalanced mechanical loading $(2,39)$. The first model we generated decreased mechanical load by partially transecting Achilles tendons in the middle of tendon body in 3-month-old male mice, termed semi-Achilles tendon transection (SMTS) (Supplemental Figure 1; supplemental material available online with this article; https://doi.org/10.1172/ JCI96186DS1). The bone volume over total tissue volume (BV/ TV) in the posterior calcaneal tuberosity (PCT), the site of Achilles tendon attachment to calcaneus, was decreased in SMTS mice compared with the sham group, as determined by $3 \mathrm{D} \mu \mathrm{CT}$ analysis 2 weeks after surgery, but restored 8 weeks after SMTS (Figure 1, A and B). The trabecular bone thickness ( $\mathrm{Tb} . \mathrm{Th}$ ), trabecular number (Tb.N), and trabecular separation (Tb.Sp) were decreased 4 weeks after SMTS. Tb.Th and Tb.N were back to levels similar to those of controls 8 weeks after SMTS, while Tb.Sp remained significantly lower in relation to sham-operated controls (Figure 1B). Moreover, a dramatically higher trabecular pattern factor (Tb.Pf), a quantitative parameter of the connectiveness and microarchitecture of trabecular bone (40), was noted in the SMTS mice compared with sham-operated controls, indicating uncoupled bone remodeling (Figure 1B). Evident bony projections at the PCT were present in 4 of 10 mice 8 weeks after SMTS (Figure 1A). The BV/TV, Tb.N, and $\mathrm{Tb}$.Th of vertebral bodies were not significantly changed in the SMTS group compared with controls at week 8 (Supplemental Figure 2). Tartrate-resistant acid phosphatase (TRAP) staining showed that the osteoclast surface per bone surface (OCS/BS) increased in PCT trabecular bone 1 and 2 weeks after surgery and was back to normal levels on week 4 after surgery (Figure 1, C and D) while leaving large bone marrow cavities (Figure 1C). A significantly higher active TGF- $\beta$ concentration in serum was seen in the SMTS mice relative to sham-operated mice from 1 to 8 weeks after 
A

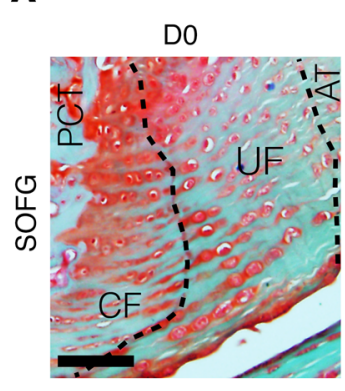

SMTS

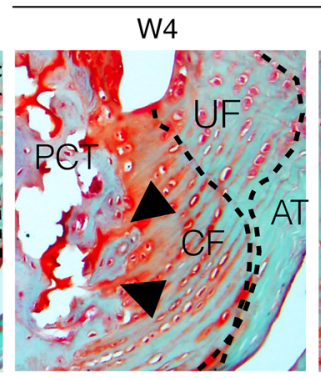

W8

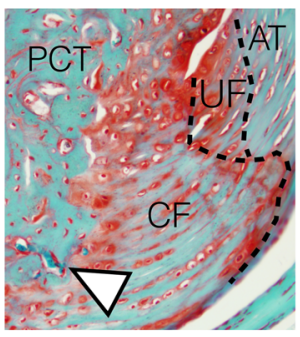

B

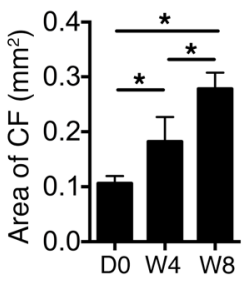

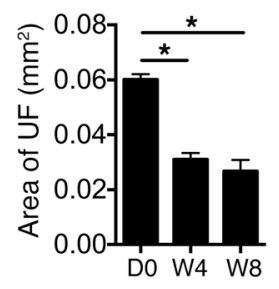

C

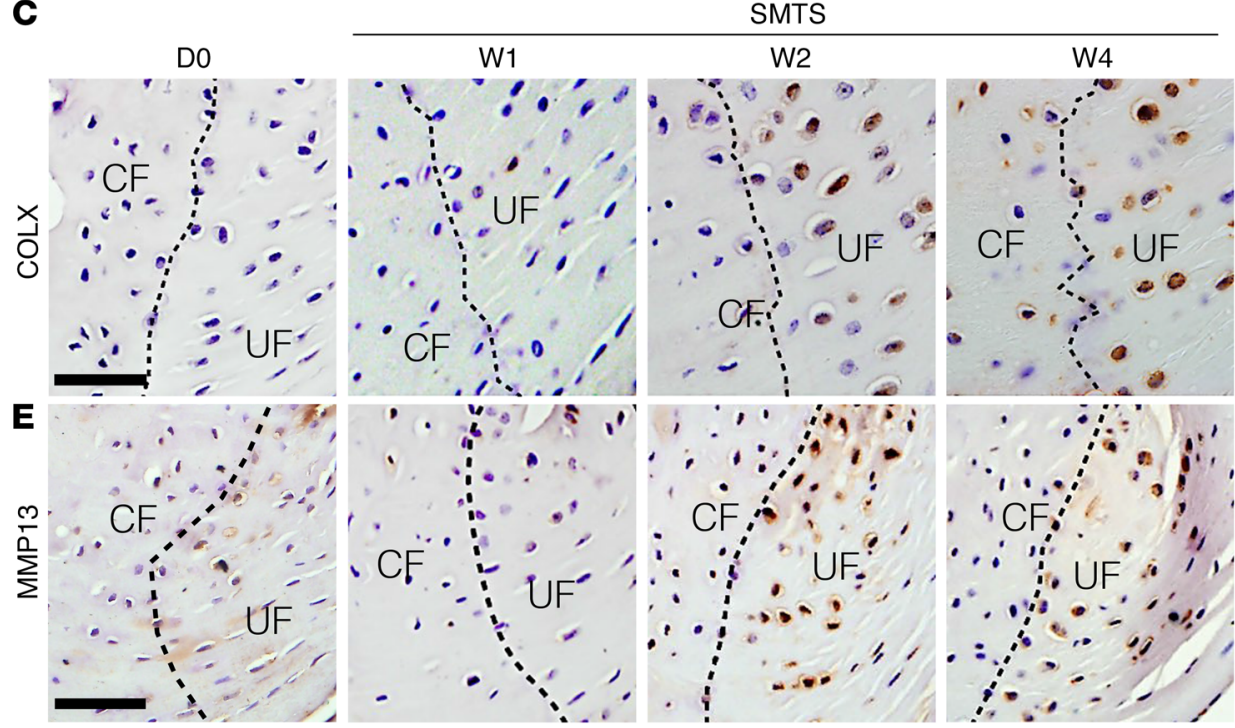

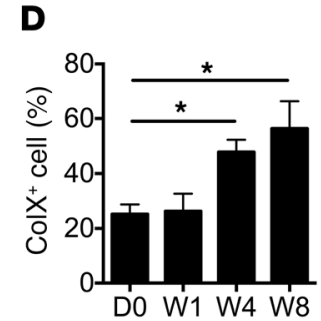

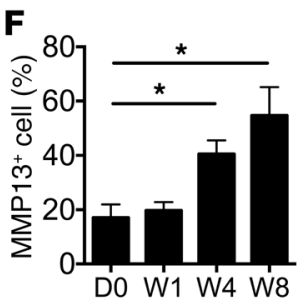

Figure 3. Fibrocartilage displays mineralization in Achilles tendon enthesis. (A) SOFG staining of Achilles tendon enthesis compartment, proteoglycan (red), bone (green) and Achilles tendon (green). Black arrowheads indicate direct connection between bone marrow and CF. White arrowhead indicates blood vessel invasion from PCT bone to CF. Scale bar: $200 \mu \mathrm{m}$. (B) Quantitative analysis of area of CF and UF. (C and E) Immunohistochemical and ( $\mathbf{D}$ and $\mathbf{F})$ quantitative analysis of (C and $\mathbf{D})$ COLX+ cells and (E and $\mathbf{F})$ MMP13+ cells (bottom) in fibrocartilage of mouse Achilles tendon enthesis after SMTS surgery. Dotted lines separate CF and UF. Scale bars: $150 \mu \mathrm{m}$. Data shown as mean \pm SEM. $n=10 .{ }^{*} P<0.05$ compared with the D0 group or between groups. AT, Achilles tendon.

surgery (Figure 1E), consistent with previous studies reporting higher TGF- $\beta$ after trauma (41-44). The total serum TGF- $\beta 1$ levels did not change significantly (Supplemental Figure 3). Immunostaining further demonstrated that the number of cells positive for phosphorylated Smad2 $/ 3\left(\mathrm{pSmad} 2 / 3^{+}\right)$, a downstream signal of TGF- $\beta$, was higher in bone marrow 1,2 , and 4 weeks after surgery as compared with that in sham-operated controls (Figure 2, $A$ and B), suggesting increased active TGF- $\beta$ levels. We compared $\mathrm{pSmad} 2 / 3$ staining to that in bone marrow cells distant from the PCT site and found no statistically significant differences between sham-operated mice and SMTS mice. Mature osteoblasts, noted by osteocalcin staining, were increased in the PCT bone marrow from 2 weeks to 8 weeks after SMTS (Figure 2, C and D), leading to a restoration in BV/TV by 8 weeks (Figure 1B). The serum osteocalcin levels were comparable to those in the controls 4 weeks after SMTS, but were significantly higher at 8 weeks (Figure 2E). Calcein double labeling confirmed that SMTS mice had more bone formation at the PCT as compared with sham-operated controls (Figure 2, F and G). We created a second Achilles enthesopathy model, termed dorsiflexion immobilization (DI), where we fixed the ankles of mice in a dorsiflexion position to mimic overuse of Achilles tendons with increased mechanical load (Supplemental Figure 4, A-E). The changes in histomorphometry parameters (BV/TV, Tb.Th, Tb.N, Tb.Sp, and Tb.Pf by $\mu \mathrm{CT}$ ) and TGF- $\beta$ signaling (pSmad2/3 staining) in the DI model were similar to those in the SMTS model, while differences in osteoclast activity $(P=$ 0.061 ) and active serum TGF- $\beta 1$ were not significantly different than those in the controls (Supplemental Figure 5, A-G). These results suggest that altered mechanical loading induces uncoupled bone resorption and formation in PCT, elevating active TGF- $\beta$ concentrations in these regions.

High levels of active TGF- $\beta$ induce fibrocartilage degeneration in SMTS mice. We next examined the fibrocartilage of Achilles tendon enthesis after SMTS. Safranin O and Fast Green (SOFG) staining showed the areas of CF increased and UF decreased in SMTS mice 4 and 8 weeks after surgery compared with those in age and sex-matched sham-operated mice, with the tidemark moving closer to the interface between UF and the Achilles tendon (Figure 3, A and B). Proteoglycan decreased in UF in the SMTS mice both 4 and 8 weeks after surgery (Figure 3A). 
A
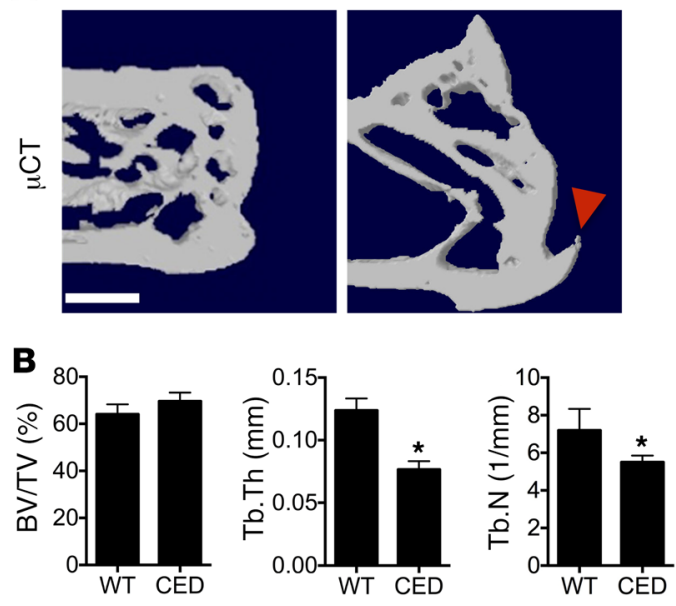

C
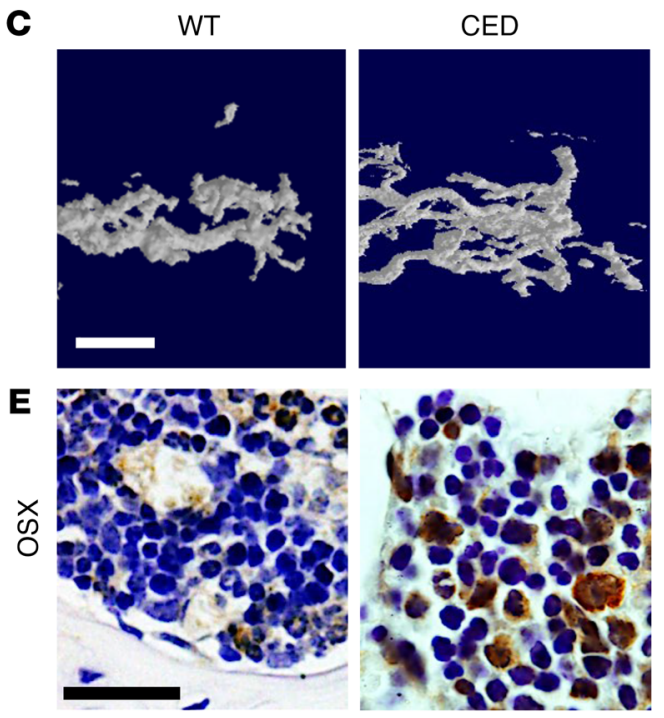

G
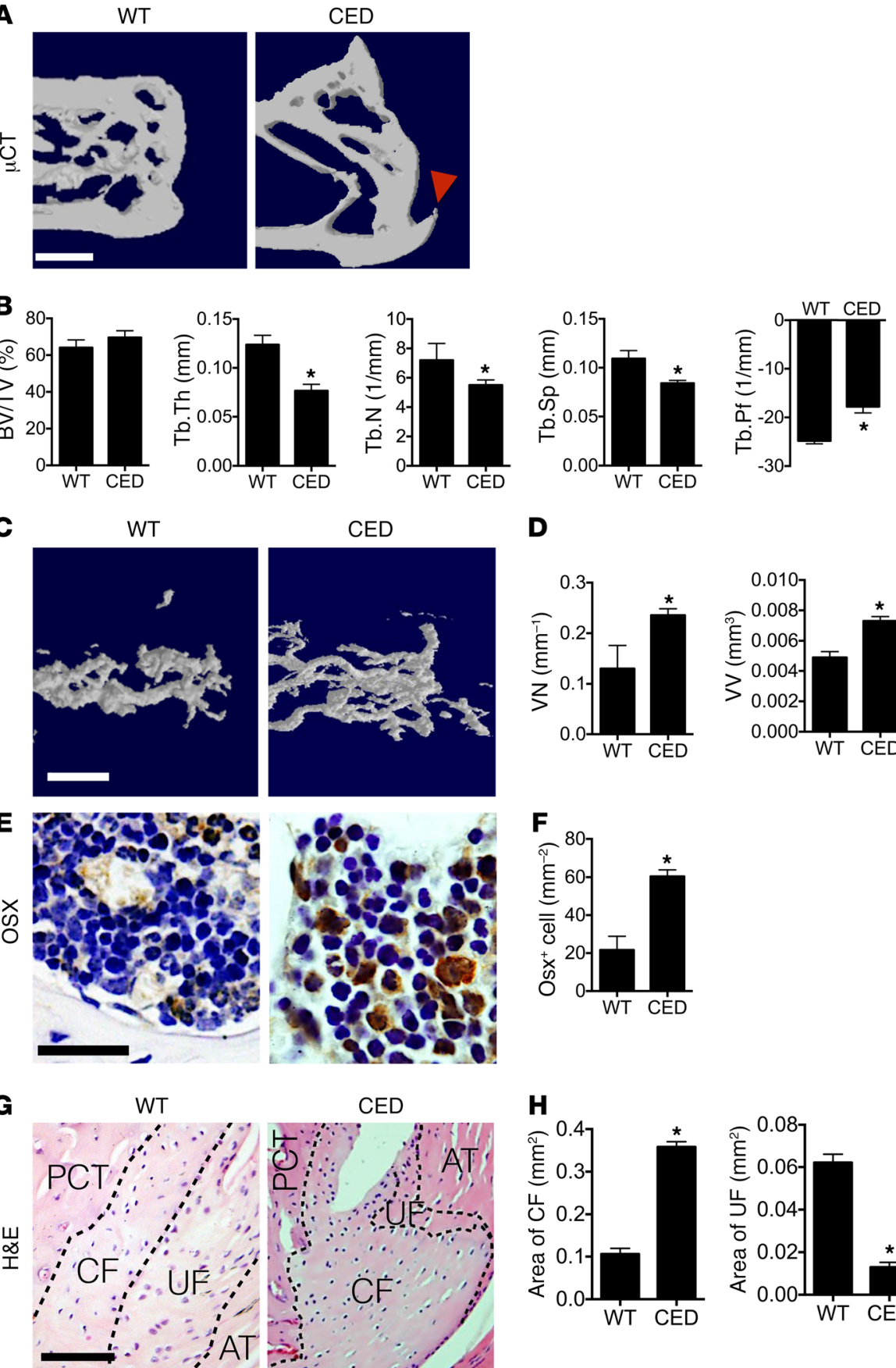

D
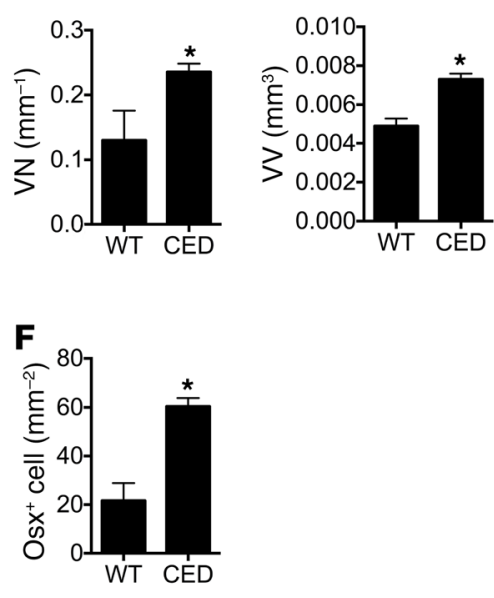

H
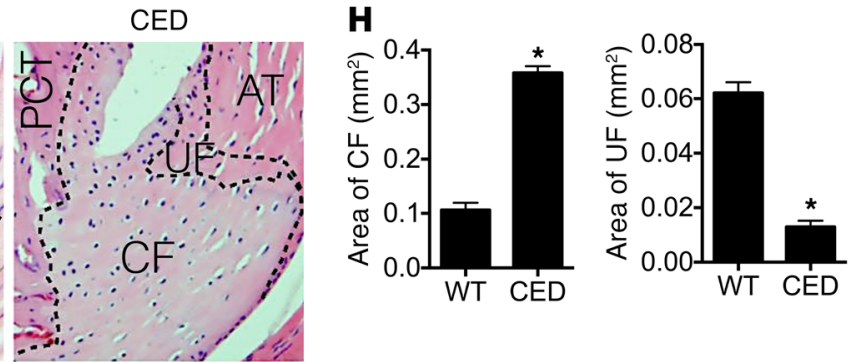

Figure 4. CED mice show an Achilles tendon enthesopathy phenotype. (A) $\mu \mathrm{CT}$ images of the PCT (sagittal view). Red arrowhead shows bony projections. Scale bar: $500 \mu \mathrm{m}$. (B) Quantitative analysis of BV/TV, Tb.Th, Tb.N, Tb.Sp, and Tb.Pf in PCT determined by $\mu \mathrm{CT}$ analysis. (C) $\mu$ CT-based microangiography of the calcaneus and (D) quantitative analysis of vessel number (VN) and vessel volume (VV). Scale bar: $100 \mu \mathrm{m}$. (E) Immunostaining and (F) quantitative analysis of $\mathrm{Osx}^{+}$(brown) cells in the PCT bone marrow of WT and CED mice. Scale bar: $50 \mu \mathrm{m}$. (G) H\&E staining of Achilles tendon enthesis. PCT bone, $C F$, and UF are separated by dotted lines. Scale bar: $200 \mu \mathrm{m}$. (H) Quantitative analysis of areas of CF and UF. (I) SOFG staining of Achilles tendon enthesis. Scale bar: $200 \mu \mathrm{m}$. Data shown as mean \pm SEM. $n=10 .{ }^{*} P<0.05$ compared between groups.
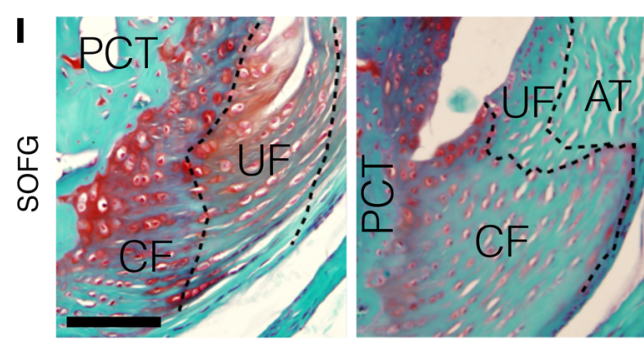

Moreover, bone marrow directly linked to CF by 4 weeks, with blood vessel invasion noted into CF in mice 8 weeks after SMTS surgery (Figure 3A). We also observed elevated concentrations of COLX (Figure 3, C and D) and MMP13 (Figure 3, E and F) in fibrocartilage in SMTS mice as compared with sham-operated controls, suggesting the process of enthesis degeneration (45, 46). The DI mouse model also revealed similar results (Supplemental Figure 6, A-F). These results suggest that unbalanced mechanical loading induces fibrocartilage degeneration in Achilles tendon enthesis. 
A
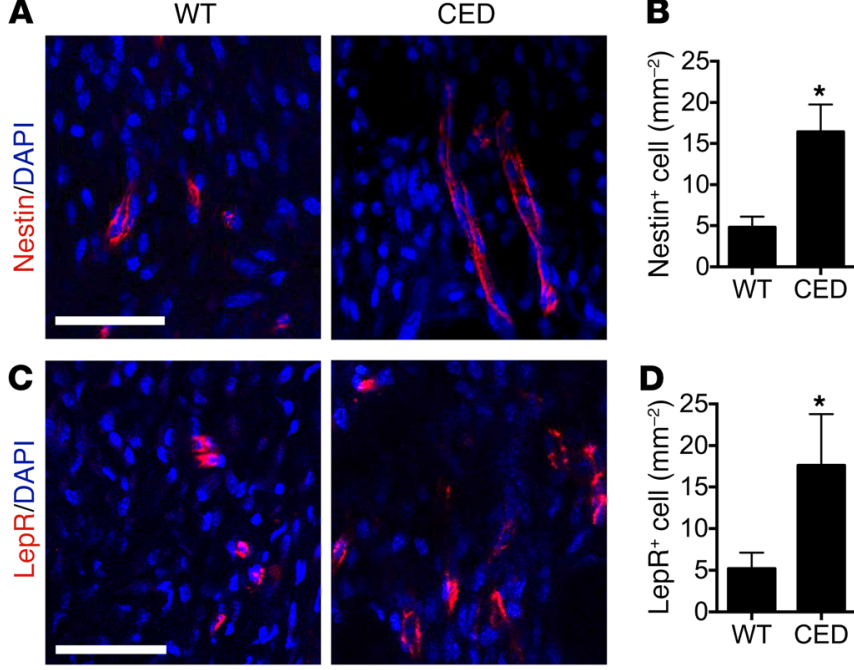

Figure 5. Nestin ${ }^{+}$and LepR $\mathrm{R}^{+}$progenitor cells are increased in HO in CED mice. (A and $\mathbf{C})$ Immunostaining and (B and $\mathbf{D})$ quantitative analysis of (A and $\mathbf{B})$ Nestin $^{+}$(red) cells and (C and $\left.\mathbf{D}\right)$ LepR ${ }^{+}$cells in the PCT bone marrow 4 weeks after sham and SMTS operations. Scale bars: $30 \mu \mathrm{m}$. Data shown as mean \pm SEM. $n=10$. ${ }^{*} P<0.05$ compared between groups.

Overexpression of active TGF- $\beta$ in bone induces enthesopathy. The SMTS and DI mouse models showed a pathology (Figures 1-3, Supplemental Figure 4, and Supplemental Figure 5) similar to that observed in human enthesopathy (11-13), implying that high concentrations of active TGF- $\beta$ may contribute to the pathogenesis of enthesopathy. To further validate the role of elevated active TGF- $\beta$ in heterotopic ossification, we determined whether high levels of TGF- $\beta$ could induce spontaneous enthesopathy with a CED mouse model. Previously, we generated this CED mouse model in which active TGF- $\beta 1$ is overexpressed in bone (36). The mice displayed abnormal bone remodeling due to uncoupling of bone formation and resorption. We examined the changes in Achilles tendon entheses in the CED mice. $\mu \mathrm{CT}$ images showed uncoupled bone formation in the PCT trabecular bone, with enlarged CF noted as a bony projection at the Achilles tendon enthesis in CED mice relative to WT littermates (Figure 4A). Quantitative analysis of $\mu \mathrm{CT}$ data revealed that CED mice exhibited increases in Tb.Pf that were similar to those observed in enthesopathy mice 8 weeks after SMTS or DI and that there were no differences in BV/TV (Figure 4B). Tb.Th, Tb.N, and Tb.Sp were significantly lower in CED mice than in their WT counterparts (Figure 4B). Angiogenesis analysis utilizing MICROFIL contrast-enhanced angiography revealed that the vessel volume and number of blood vessels in PCT bone marrow were significantly higher in CED mice compared with WT controls (Figure 4, C and D). The number of osterix positive $\left(\mathrm{Osx}^{+}\right)$osteoprogenitors/osteoblasts was also significantly higher in the PCT bone marrow of CED mice compared with WT controls (Figure 4, E and F), indicating that increased osteoblastic differentiation likely contributed to de novo bone formation. We found the area of the $\mathrm{CF}$ layer was greater in the CED mice, whereas the UF area was less (Figure 4, G and H). Compared with WT littermates, there was also significantly lower proteoglycan expression in the CF and UF zones in the CED mice (Figure $4 \mathrm{I})$. Therefore, overexpression of active TGF- $\beta$ in bone recapitulated the enthesopathy phenotype in SMTS mice.
We previously found that high concentrations of TGF- $\beta 1$ induced the formation of $\mathrm{Nestin}^{+}$cell clusters, leading to formation of bone marrow osteoid islets in the progression of osteoarthritis (36). In adult bone marrow, Nestin ${ }^{+}$cells have been shown to represent a subset of bone marrow precursor cells mainly in endothelial cell lineage and mesenchymal lineage (47-49). We also determined whether there were increased stem/progenitor cells in PCT bone marrow during the enthesis degeneration in CED mice. We detected clusters of Nestin ${ }^{+}$cells (Figure 5, A and B) and leptin receptor-positive (LepR $\mathrm{R}^{+}$) cells (Figure 5, C and D), which are major osteoblast-forming mesenchymal stromal precursor cells (MSPCs) in adult bone marrow (50), in the calcaneal bone marrow right at the edge of the fibrocartilage zone in CED mice, but not in WT littermates. The results suggest that TGF- $\beta$-recruited endothelial progenitor cells (EPCs) and MSPCs may contribute to the excessive angiogenesis and ossification during enthesis degeneration. Therefore, CED mice have an enthesopathy phenotype similar to that of SMTS and DI mouse models, suggesting that high concentrations of active TGF- $\beta 1$-induced abnormal bone formation and vessel formation contribute to Achilles tendon enthesopathy.

Systemic injection of TGF- $\beta$ antibody attenuates progression of enthesopathy. As SMTS, DI, and CED mice showed similar pathological changes, we utilized the SMTS model only for the following experiments. Injection of a TGF- $\beta$-neutralizing antibody (1D11) has been shown to rescue uncoupled bone formation induced by high concentrations of active TGF- $\beta 1$ (35). We therefore investigated the effects of TGF- $\beta$ inhibition on enthesopathy pathogenesis. First, we screened different doses of 1D11 in experimental mice to identify the optimal dose and injection frequency, which was 5 mg per kg body weight injected weekly (Supplemental Figure 7, A and B). Vehicle antibody (13C4) consisted of an identical IgG complex lacking any TGF- $\beta$-binding capabilities. 1D11 significantly decreased the concentration of active TGF- $\beta 1$ in whole blood in SMTS mice relative to vehicle (Supplemental Figure 8A). TGF- $\beta$ signaling was also reduced at the tissue level, as demonstrated by immunostaining of cells positive for $\mathrm{pSmad} 2 / 3$ in the fibrocartilage in Achilles tendon enthesis (Supplemental Figure 8, B and C) as well as in the PCT bone marrow in the SMTS 1D11-treated mice relative to SMTS vehicle-treated mice (Supplemental Figure 8, D and E). $\mu \mathrm{CT}$ analysis of the PCT in 1D11-treated SMTS mice demonstrated increased PCT BV/TV, Tb.Th, Tb.N, and Tb. Sp and decreased Tb.Pf relative to vehicle-treated SMTS mice at 4 weeks (Figure 6, A and B). There were no statistically significant differences in these parameters comparing the SMTS 1D11-treated mice relative to the controls, sham-operated mice treated with either vehicle or 1D11, demonstrating the prevention of changes in trabeculae microarchitecture and connectivity (Figure 6A). We also examined the inflammatory cytokines TNF- $\alpha$ and IL- $1 \beta$ and found neither vehicle- nor antibody-treated mice showed high levels of expression (Supplemental Figure 9). The increase in Nestin ${ }^{+}$cells observed in the SMTS vehicle-treated mice relative to sham-surgery controls was attenuated by 1D11 (Figure 6, C and D). Similarly, the number of $\mathrm{CD} 31^{+}$vessels, a marker of endothelial cells, was also significantly higher in the PCT bone marrow of vehicletreated SMTS mice relative to sham-surgery controls, and this effect was reduced by 1D11 treatment (Figure 6, E and F). Injection of 1D11 also significantly decreased $\mathrm{Osx}^{+}$cell numbers in 
A
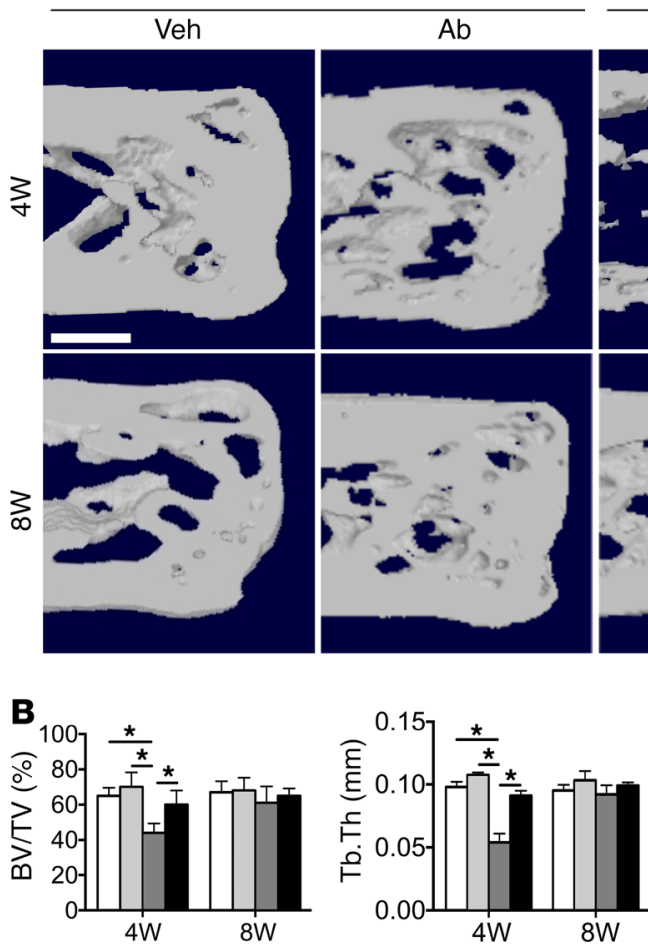

SMTS
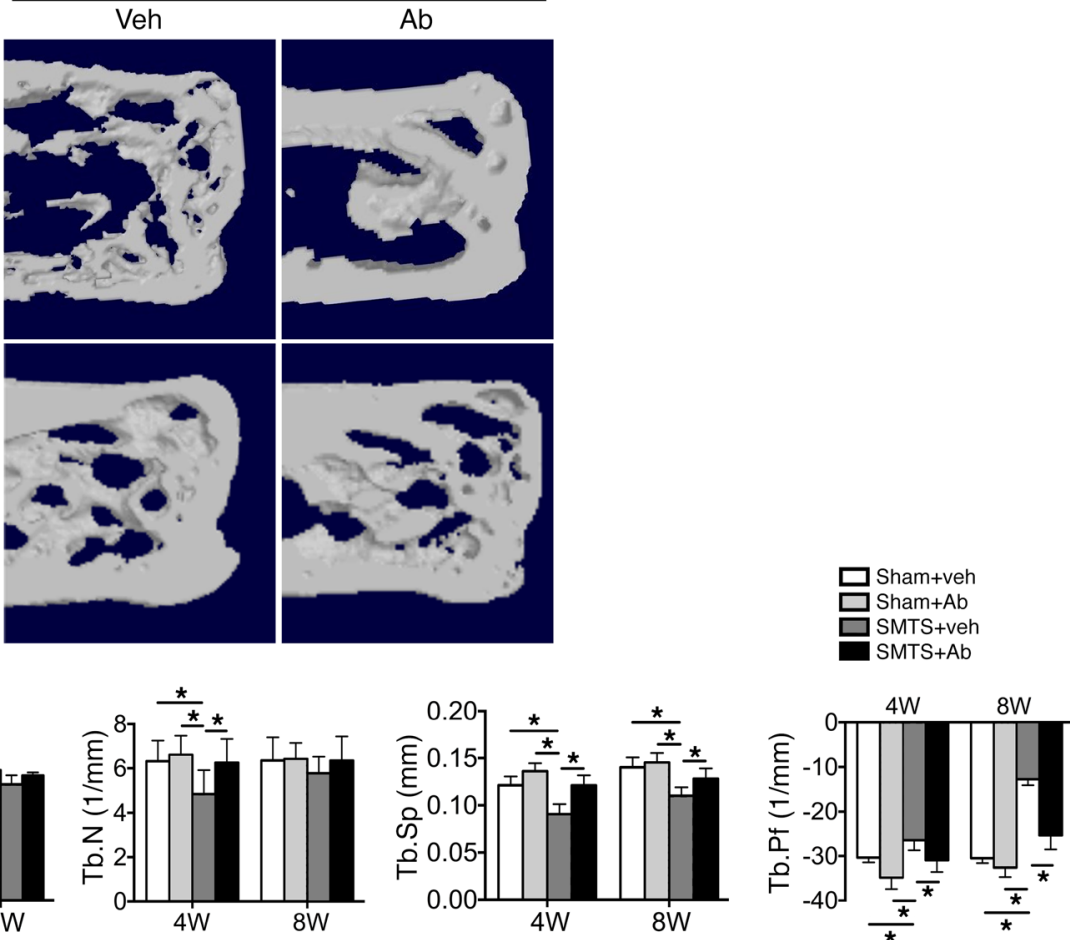

C
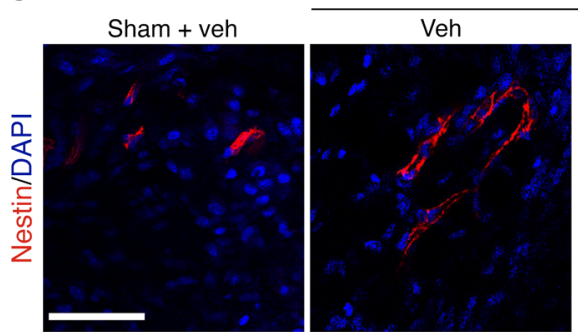

SMTS 4 W
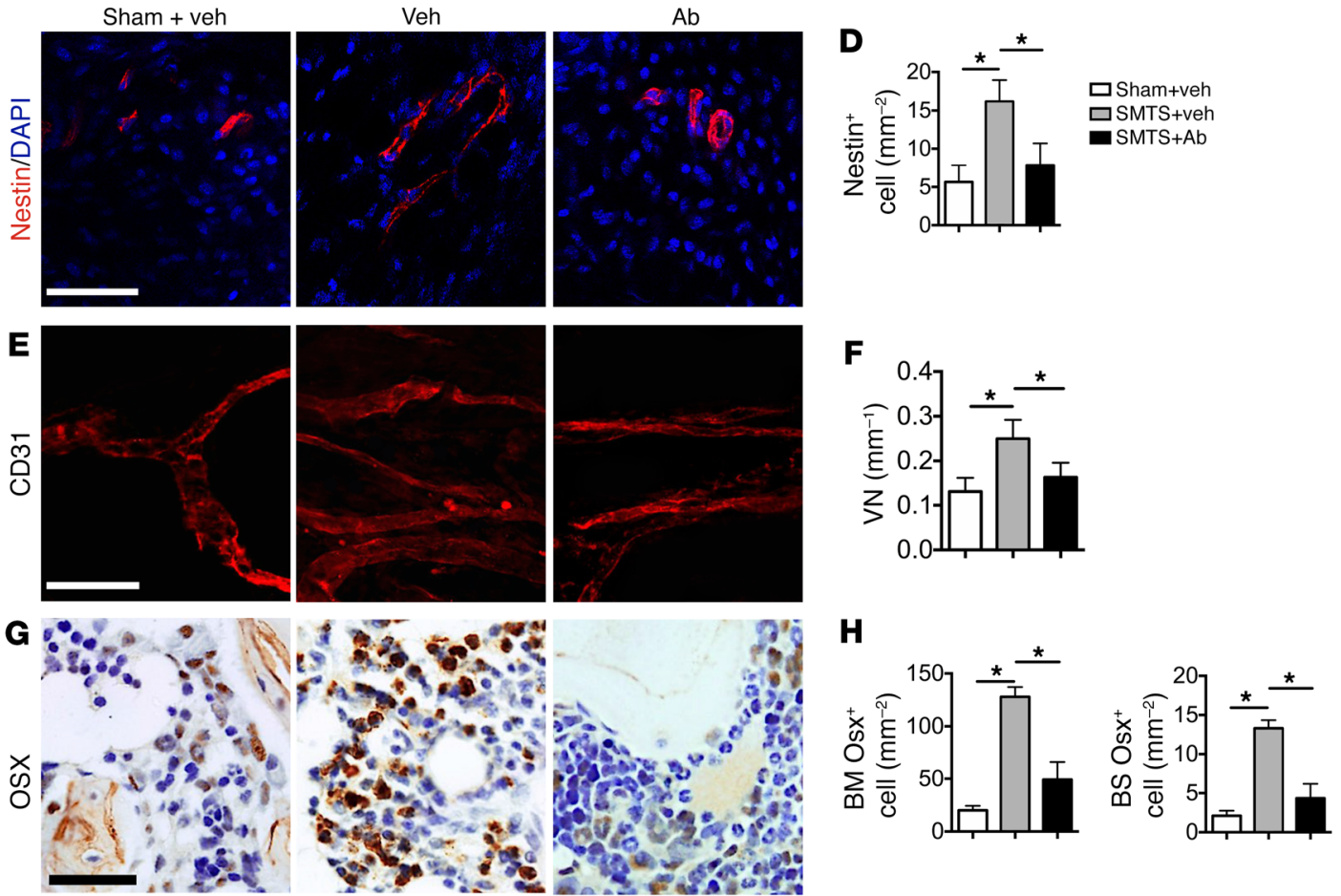

Figure 6. Systemic injection of TGF- $\beta 1$ antibody maintains Achilles tendon enthesis structure and reduces the unregulated TGF- $\beta$ signaling. (A) $\mu$ CT images of the PCT (sagittal view) of mice treated with $5 \mathrm{mg}$ per kg body weight of the TCF- $\beta$-neutralizing antibody $1 \mathrm{D} 11$ weekly for 30 days and analyzed 4 or 8 weeks after SMTS or sham surgery. Scale bar: $500 \mu \mathrm{m}$. (B) Quantitative analysis of BV/TV, Tb.Th, Tb.N, Tb.Sp, and Tb.Pf in PCT determined by $\mu$ CT analysis. (C) Immunostaining and (D) quantitative analysis of Nestin ${ }^{+}$cells (red) in the PCT bone marrow. Scale bar: $30 \mu \mathrm{m}$. (E) Immunostaining of CD31+ (red) vessels and the (F) quantification of the number of vessels positive for CD31 (per mm²). Scale bar: $100 \mu \mathrm{m}$. (C) Osx ${ }^{+}$cells (brown) in the PCT and (H) quantifications of Osx ${ }^{+}$cell number in PCT bone marrow and on PCT BS. Data shown as mean \pm SEM. $n=10$. ${ }^{*} P<0.05$ compared between groups or to the sham group. Veh, vehicle. 
A

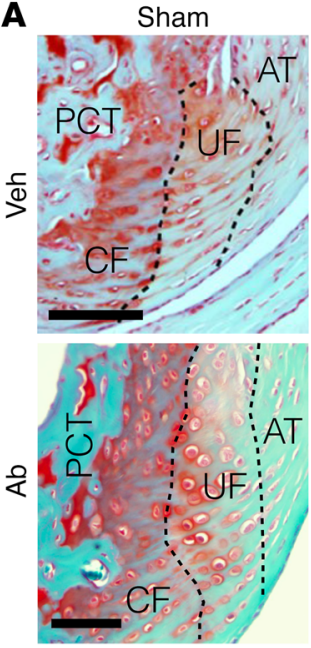

SMTS $4 \mathrm{~W}$

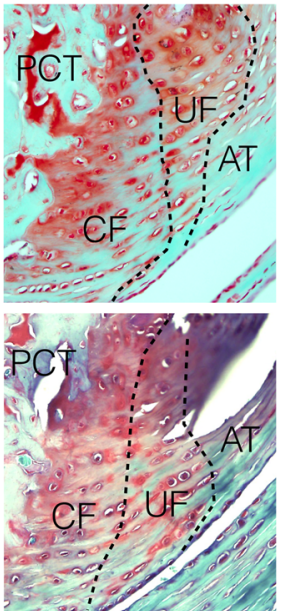

SMTS $8 \mathrm{~W}$
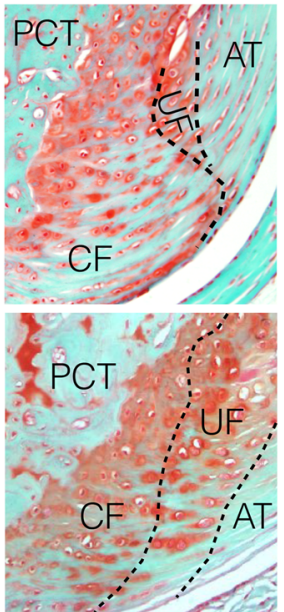

B

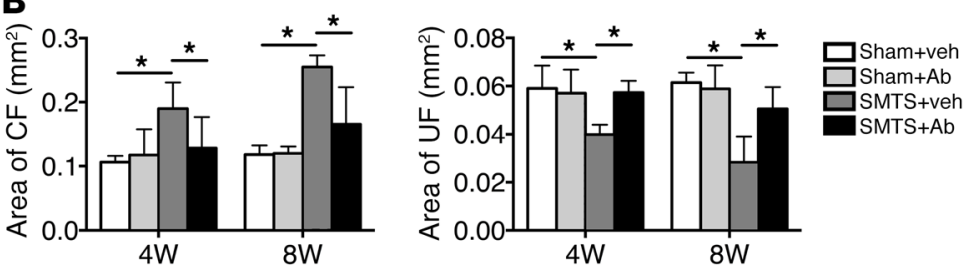

SMTS $4 \mathrm{~W}$

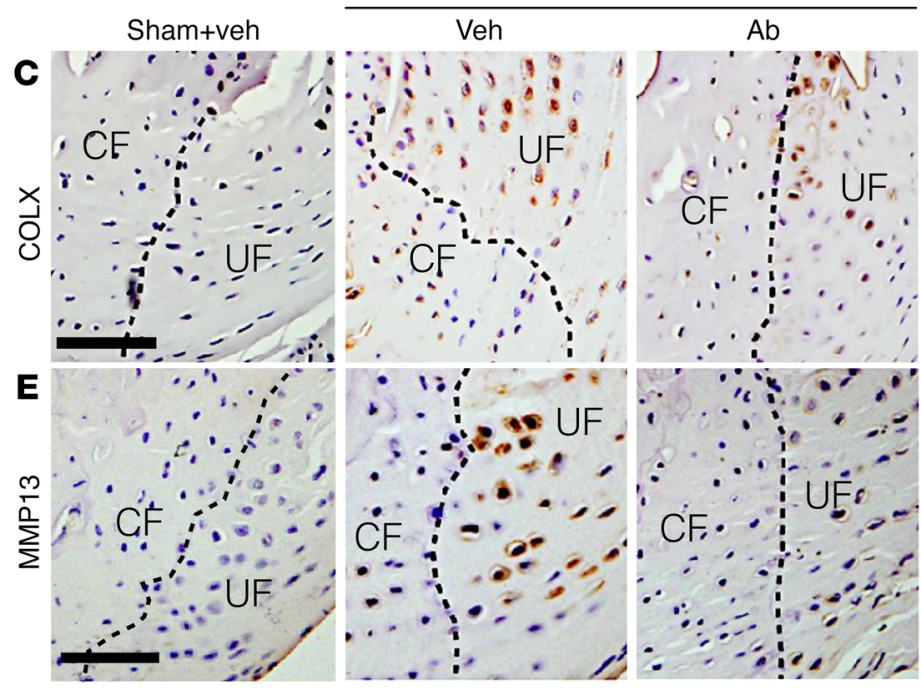

D

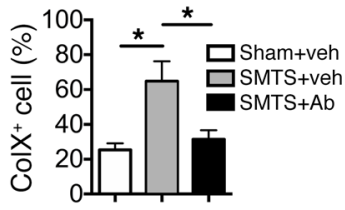

F

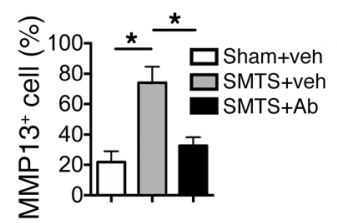

SMTS mice relative to vehicle. Furthermore, $\mathrm{Osx}^{+}$cells were noted largely in the bone marrow of SMTS vehicle-treated mice, whereas the Osx ${ }^{+}$cells in the SMTS 1D11 mice were largely located on the BS, similarly to sham-operated controls (Figure 6, G and H). Collectively, these results indicate that inhibition of TGF- $\beta$ signaling prevents increases in Nestin ${ }^{+}$cells after surgery that may contribute to osteogenesis and vessel formation.

TGF- $\beta$ antibody attenuates fibrocartilage destruction in enthesopathy. As the PCT bone phenotype was improved with 1D11 in the SMTS mice, we next evaluated the effect of 1D11 on the enthesis in this mouse model. The changes in the areas of CF and UF caused by SMTS were restored in SMTS 1D11 mice relative to sham-operated mice, as revealed by SOFG staining (Figure 7, A and B). Furthermore, 1D11 also attenuated the elevated expres-
Figure 7. Systemic injection of TGF- $\beta$ antibody attenuates enthesis changes in enthesopathy mice. (A) SOFG staining of Achilles tendon enthesis. PCT, CF, UF, and Achilles tendon ( $T$ ) are separated by dotted lines. Scale bars: $200 \mu \mathrm{m}$. (B) Quantitative analysis of areas of CF and UF. (C and $\mathbf{E}$ ) Immunohistochemical (staining) and ( $\mathbf{D}$ and $\mathbf{F}$ ) quantitative (bar chart, right) analysis of (C and $\mathbf{D})$ COLX+ cells and ( $E$ and $\mathbf{F}$ ) $\mathrm{MMP}^{+} 3^{+}$cells (bottom) in fibrocartilage (CF and UF are separated by dotted lines) of mouse Achilles tendon enthesis after SMTS surgery. Scale bars: $150 \mu \mathrm{m}$. Data shown as mean \pm SEM. $n=10$. ${ }^{*} P<0.05$ compared between groups or to the sham group. sion of MMP13 or COLX in fibrocartilage as compared with SMTS vehicle treatment (Figure 7, C-F). Collectively, these results indicate that systemic inhibition of TGF- $\beta 1$ activity maintained enthesis and fibrocartilage structure.

Genetic knockout Tgfbr2 in MSCs prevents enthesopathy. To determine the role of Nestin ${ }^{+}$cells in enthesopathy, we crossed Nestin-creERT2 mice with R26R-EYFP mice to generate tamoxifen-inducible Nestin-creERT2::YFP mice to trace $\mathrm{Nestin}^{+}$cells during enthesopathy progression. Nestin ${ }^{+}$cells did not target $\mathrm{CF}$ or UF directly (Supplemental Figure 10). Coimmunostaining of CD31/yellow fluorescent protein (YFP) and endomucin (Emcn)/ YFP in PCT bone marrow revealed that $60 \%$ of Nestin lineage cells participated in $\mathrm{CD} 31^{+}$vessel formation and $\mathrm{Emcn}^{+}$vessel formation (Figure 8, A and B). This suggests that bone marrow Nestin 

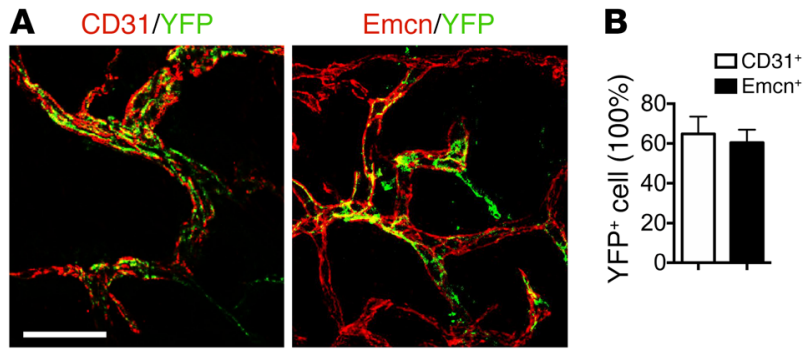

Figure 8. Nestin+ cells gives rise to vessels in PCT bone marrow after SMTS. (A, left) $\mathrm{CD} 1^{+}$cells (red), $\mathrm{YFP}^{+}$cells (green), and (A, right) $\mathrm{Emcn}^{+}$ cells (red), YFP+ cells (green). (B) Quantitation in PCT bone marrow of Nestin-creERT2::R26R-EYFP mice 8 weeks after SMTS. Scale bar: $100 \mu \mathrm{m}$ $n=10$ per group. Data shown as mean \pm SEM.

lineage cells participated in osteogenic CD $31^{\text {hi }} \mathrm{Emcn}^{\text {hi }}$ type $\mathrm{H}$ vessel (51) formation in enthesopathy. In contrast, no Nestin lineage cells were detected in the tendons adjacent to Achilles tendon enthesis, while abundant $\mathrm{CD} 1^{+} \mathrm{YFP}^{+}$vessels and $\mathrm{Emcn}^{+} \mathrm{YFP}^{+}$vessels were formed in the tendon semitransection site (Supplemental Figure 11, A and B).

To determine whether the changes in the enthesis were the sequela of elevated Nestin ${ }^{+}$cells by high levels of bone marrow TGF- $\beta$-signaling events, we employed another inducible mouse model genetically targeting TGF- $\beta$ signaling in Nestin ${ }^{+}$cells. We injected Nestin-CreERT2::Tgfbr $2^{f / f l}$ mice with tamoxifen to specif-

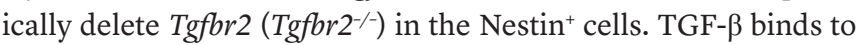
type II TGF- $\beta$ receptor (Tgfbr 2 ) and to type I TGF- $\beta$ receptor (Tgfbr1) complex to induce phosphorylation of downstream Smad2/3. Deletion of Tgfbr 2 blocks the TGF- $\beta$-signaling cascade. The microstructure of the PCT was maintained and bony projections were not noted in $\mathrm{Tg} f b r 2^{-/-}$mice, while these were evident in control SMTS Tgfbr $2^{f / f}$ mice (Figure 9A). BV/TV were decreased while Tb.Pf was increased in SMTS Tgfbr $2^{f / f}$ mice relative to sham-operated controls (Figure 9B). In contrast, SMTS Tgfbr2 $2^{-1-}$ mice showed improvement in BV/TV and Tb.Pf relative to SMTS Tgfbr $2^{f / f}$ mice. There were no statistically significant differences between SMTS Tgfbr $2^{-1-}$ mice at 8 weeks and any other treatment group, suggest-

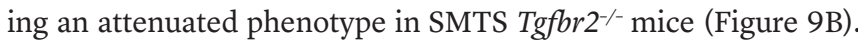
Nestin-creERT2 mice treated with tamoxifen were also used as control. No significant differences in histomorphometric parameters were found in the Nestin-creERT2 mice treated with tamoxifen compared with those in Nestin-creERT2::Tgfbr $2^{\mathrm{f} / \mathrm{f}}$ mice treated with vehicle (Supplemental Figure 12, A and B). The number of Nestin $^{+}$cells and $\mathrm{Osx}^{+}$osteoprogenitors/osteoblasts in the PCT bone marrow of SMTS Tgfbr2-/-mice were decreased, and the $\mathrm{Osx}^{+}$ cells resided primarily on the BS compared with SMTS Tgfbr $2^{f / f}$ littermates (Figure 9, C-F). Angiography analyses demonstrated that the number of blood vessels and vessel volume in PCT of SMTS Tgfbr $2^{f / f}$ mice were significantly higher relative to those of sham-surgery controls and SMTS Tgfbr2 ${ }^{-/-}$mice (Figure 9, G and $\mathrm{H})$. Deletion of Tgbfr2 in Nestin lineage cells was sufficient to prevent the change in area observed in CF and UF in SMTS mice (Figure 10, A and B). Immunostaining revealed that the expression of MMP13 and COLX was significantly attenuated in SMTS $\mathrm{Tg}_{\mathrm{fbr}} \mathrm{2}^{-/-}$mice compared with SMTS Tgfbr2 $2^{\mathrm{f} / f}$ littermates, indicat- ing the inhibition of fibrocartilage degeneration and mineralization (Figure 10, C-F). These data confirm that high concentrations of active TGF- $\beta$ contribute to the pathogenesis of Achilles tendon enthesopathy, which can be partially prevented by inhibition of TGF- $\beta$-signaling in MSCs.

\section{Discussion}

Tendon injuries occur most commonly from excessive or unbalanced strain, such as work-related injuries or sports-related injuries, and therefore affect both young and old adults $(2,39)$. In this study, we focused on pathological changes in the enthesis, as onefourth of tendon rupture happens in the enthesis and this is the most difficult condition to treat $(52,53)$.

TGF- $\beta$ plays an essential role in cartilage and bone homeostasis and relies on precise spatial and temporal activation $(31,54)$. It is expressed as an inactive latent form and deposited in the ECM in mammals (55). TGF- $\beta$ isoforms are broadly expressed in bone, cartilage, and tendon. In the bone-remodeling microenvironment, activation of bone matrix TGF- $\beta 1$ leads to migration of MSCs for osteogenesis (31). In cartilage, TGF- $\beta$ stimulates the production of ECM proteins and prevents terminal differentiation of chondrocytes $(56,57)$. In tendons, TGF- $\beta$ influences the direction of the enthesis fibrocartilage, aligning with compressive forces $(58,59)$. Disruption of the precise temporal and spatial activation of TGF- $\beta$ contributes to the pathology. Our previous study showed that high concentrations of active TGF- $\beta 1$ in subchondral bone increased the number of MSCs and osteoprogenitors to disrupt the joint microarchitecture and contributed to the pathogenesis of osteoarthritis (36). Injection of TGF- $\beta$-neutralizing antibody has been shown to improve several skeletal diseases in animal models, such as CED, osteoarthritis, and osteogenesis imperfecta (31-35). Excessive release of active TGF- $\beta$ has also been found to contribute to tendon and cartilage pathology, leading to massive tenocyte death (60) and cartilage degeneration (61). A more recent study revealed that TGF- $\beta$, released from the BS as a result of metastasis-induced bone destruction, mediates muscle weakness (62), suggesting crosstalk among adjacent tissues. In the current study, we found the excessive activation of TGF- $\beta$ also contributes to enthesopathy.

In healthy fibrocartilaginous enthesis, such as the Achilles tendon, 4 layers of transitional zone act as a functional unit (1). The 4 transitional zones confer a smooth transfer of load by giving rise to graded tissue mechanical properties (1). Semitransection of the Achilles tendon disrupts the force transmission while leaving the enthesis intact. In the current study, we found that uneven mechanical force on the Achilles tendon entheses increased bone resorption, as demonstrated by an increase in the TRAP staining in the PCT bone as early as 7 days after surgery in both the SMTS mouse models. The increased osteoclastic activity was associated with activation of TGF- $\beta$, increased Nestin ${ }^{+}$cell numbers, and subsequent excessive bone formation and angiogenesis. At the same time, degenerative changes were noted in the enthesis, revealed by increased CF area, decreased UF area, and high expression of MMP13 and COLX in the fibrocartilage. The role of excessive active bone marrow TGF- $\beta$ in enthesopathy was affirmed utilizing the CED mice. Overexpression of active TGF- $\beta$ in bone in CED mice recapitulated the enthesopathy phenotype in SMTS and DI mice, further suggesting that high concentrations 
A

Sham 8W

SMTS 8W
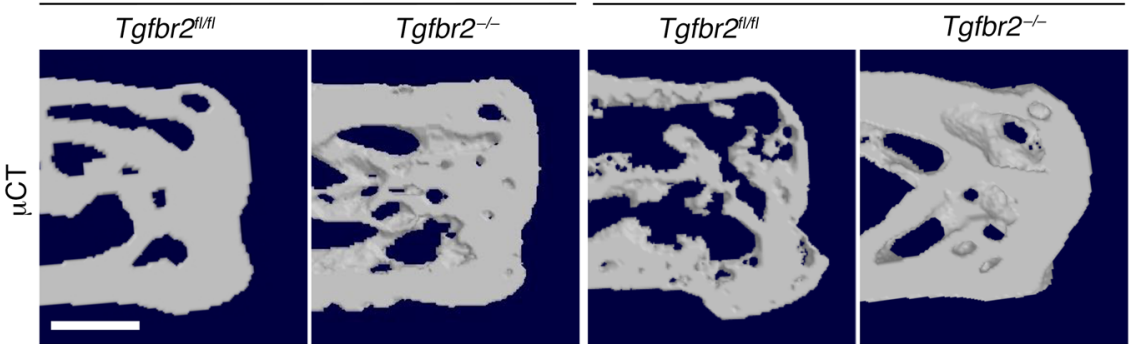

B

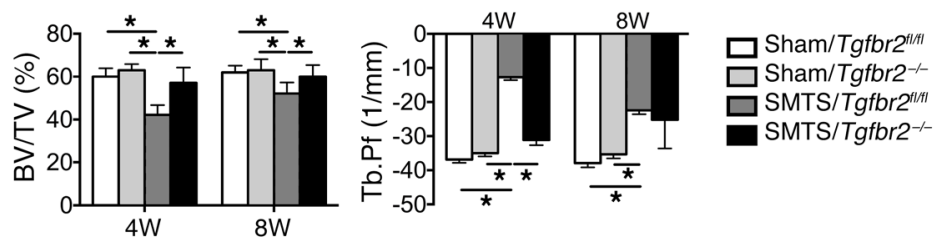

C

Sham 4W

SMTS 4W
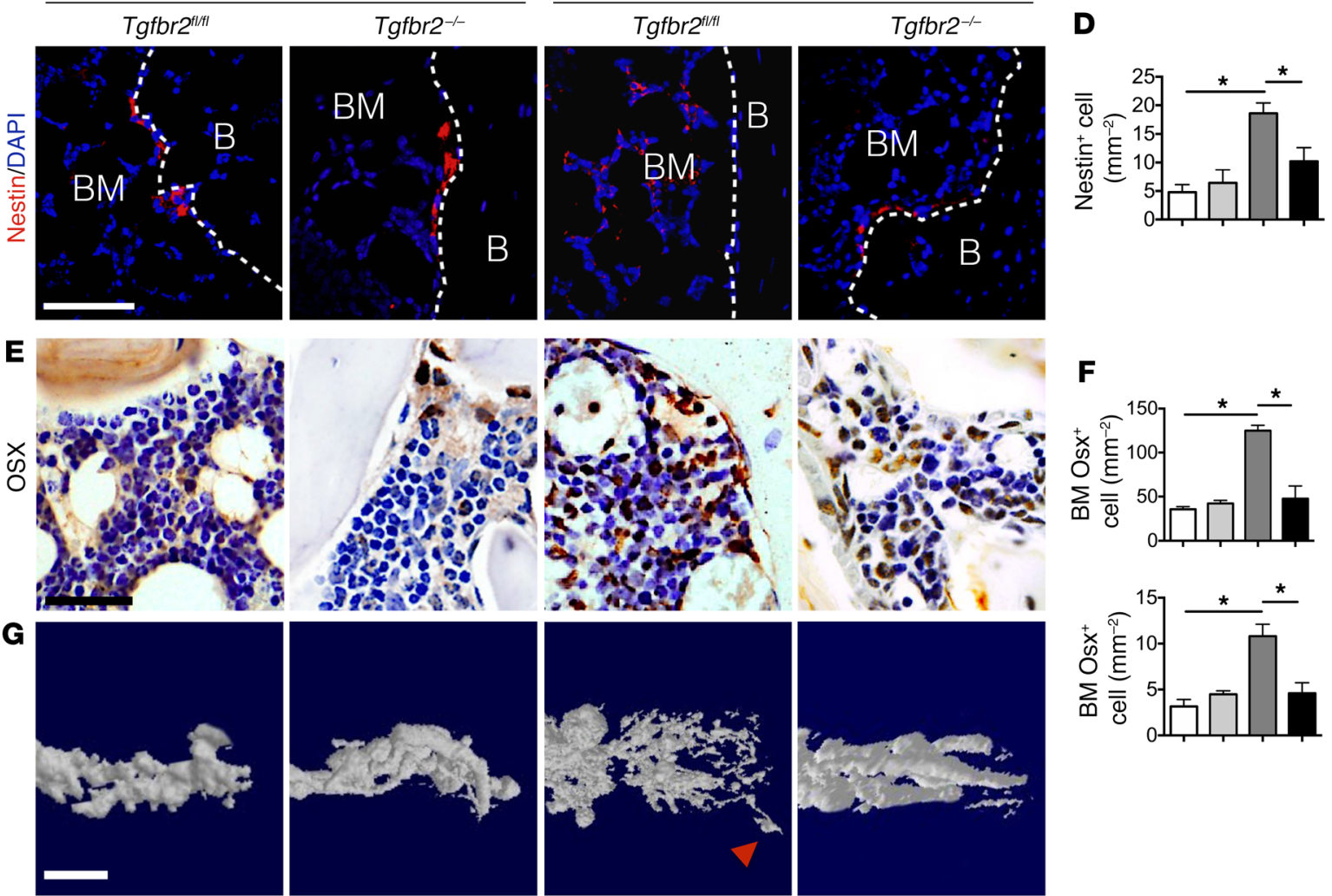

G
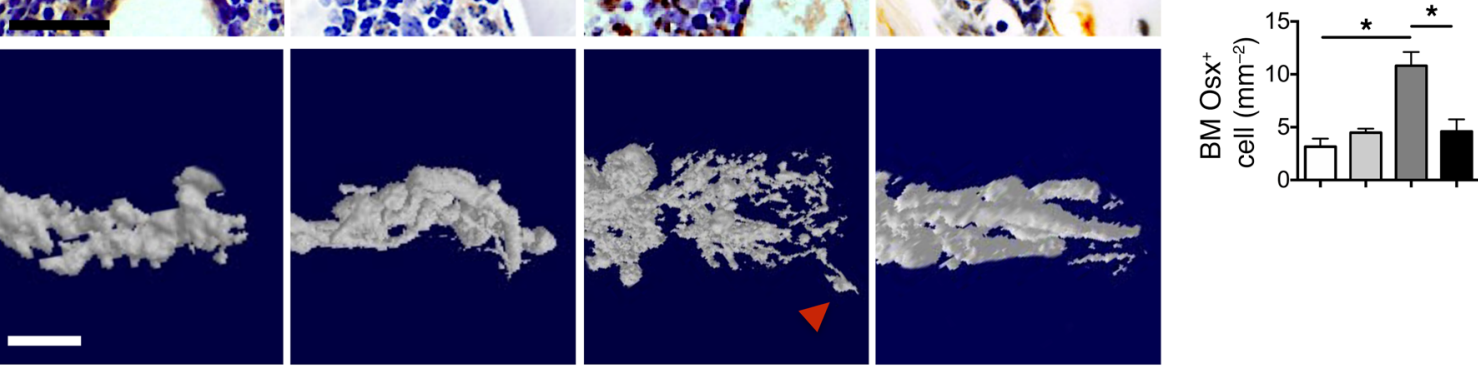

H

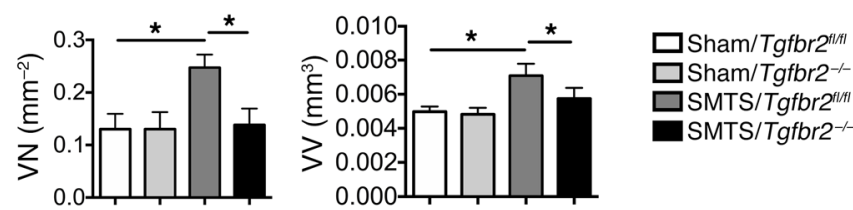

Figure 9. Genetic knockout of Tgfbr2 in Nestin+ cells results in less change in Achilles tendon enthesis after SMTS. (A) $\mu$ CT images of the PCT (sagittal

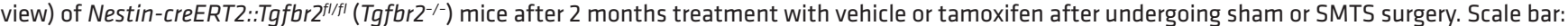
$500 \mu \mathrm{m}$. (B) Quantitative analysis of structural parameters of PCT by $\mu C T$ analysis. (C) Nestin ${ }^{+}$(red) and (E) Osx (brown) cells in the PCT bone marrow treated with vehicle or tamoxifen 4 weeks after sham and SMTS operations. White dotted lines (C) indicate bone surface. B, bone. Scale bar: $50 \mu \mathrm{m}$. (D and F) Quantifications of the number of bone marrow cells positive for Nestin and Osx in PCT bone marrow and PCT BS (per mm²). (C) $\mu$ CT-based microangiography of the calcaneus and (H) quantitative analysis of VN and VV. Red arrowhead indicates blood vessel invasion into the enthesis. Scale bar: $150 \mu \mathrm{m}$. Data shown as mean \pm SEM. $n=10 .{ }^{*} P<0.05$ compared between groups or to the sham group. 
A

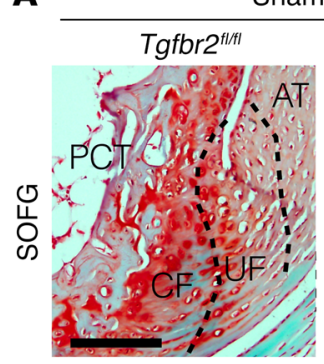

Sham 8W

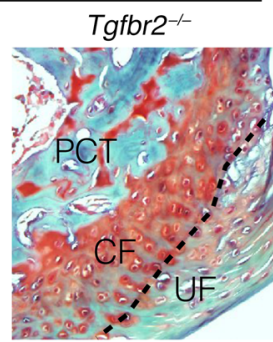

SMTS 8 W

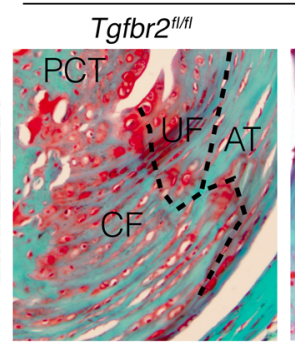

Tgfbr2 $^{-1}$

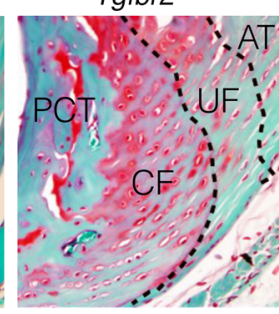

B
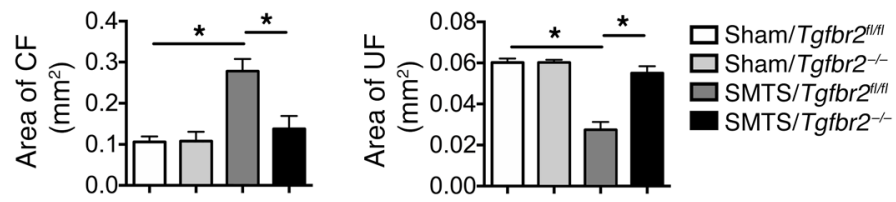

C
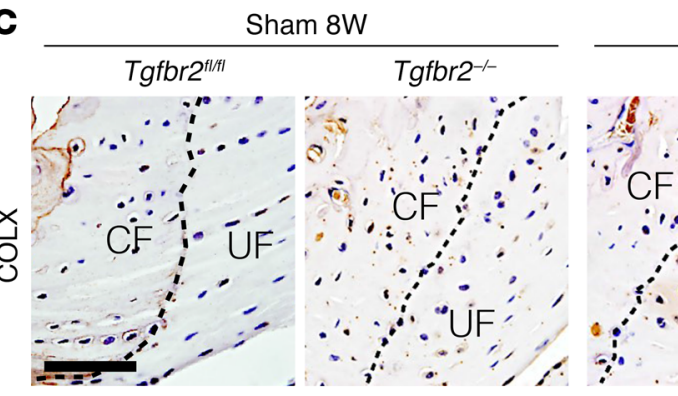

SMTS $8 \mathrm{~W}$
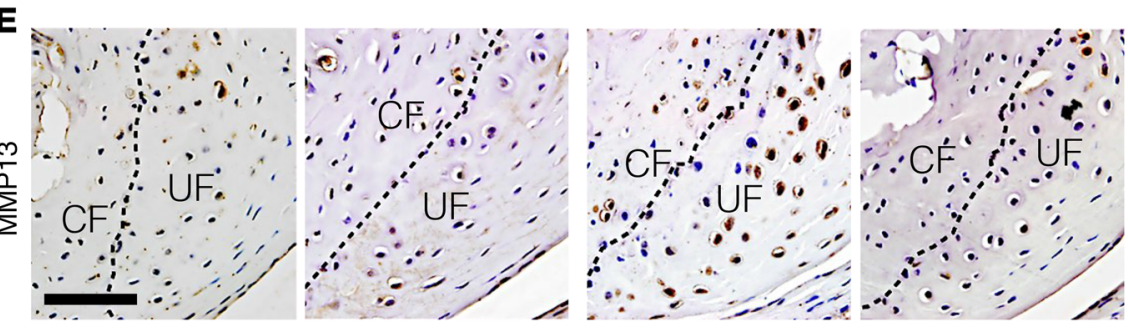

$\mathbf{F}$

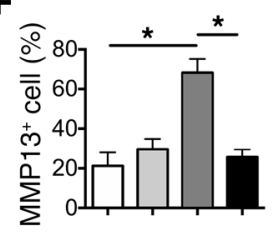

Figure 10. Deletion of Tgfbr2 in Nestin+ cells mitigates enthesis degradation in enthesopathy mice. (A) SOFC staining of Achilles tendon enthesis. PCT, CF, UF, and Achilles tendon are separated by dotted lines. Scale bar: $200 \mu \mathrm{m}$. (B) Quantitative analysis of area of CF and UF. (C and E) Immunohistochemical and ( $\mathbf{D}$ and $\mathbf{F})$ quantitative analysis of ( $\mathbf{C}$ and $\mathbf{D}) \operatorname{COLX}{ }^{+}$and $(\mathbf{E}$ and $\mathbf{F}) \mathrm{MMP13}^{+}$cells in enthesis fibrocartilage. Scale bars: $150 \mu \mathrm{m}$. Data shown as mean \pm SEM. $n=10 .{ }^{*} P<0.05$ compared between or to the sham group.

of active TGF- $\beta$ induced abnormal bone formation and vessel formation, contributing to Achilles tendon enthesopathy. Localized mineralization of Achilles tendon at the semitransection site (in the middle of tendon body) (63) likely contributes to high levels of TGF- $\beta 1$ until 8 weeks. However, we found that high levels of active TGF- $\beta$ in serum did not alter bone turnover at a distant site, such as vertebral bodies, contralateral (uninjured) SMTS enthesis, or inflammatory cytokines. Furthermore, the excessive TGF- $\beta$ was only activated in the PCT bone marrow rather than bone marrow distant from PCT in calcaneus.

We found that inhibition of TGF- $\beta$ activity was effective in preventing changes in the PCT microarchitecture and enthesis degeneration. Similar to our findings in osteoarthritis, where microarchitectural changes in subchondral bone led to articular cartilage degeneration, the deletion of Tgfb2r in Nestin lineage cells after SMTS preserved the PCT integrity with no statistically significant differences relative to sham groups. Our data also demonstrated that TGF- $\beta$ antibody treatment involves not only inhibition of Smad phosphorylation, but also a marked drop in Nestin $^{+}$cell number as well as bone and vessel formation. Recent studies demonstrate $\mathrm{Nestin}^{+}$cells can give rise to endothelial cells and mesenchymal lineage cells (47-49). The decrease in Nestin ${ }^{+}$ cell numbers in the TGF- $\beta$ antibody SMTS mice are consistent with our prior data (31) regarding the role of TGF- $\beta$ in MSC migration and likely explain the decrease in vessel and bone formation. Although effective attenuation of the progression of enthesopathy and decreased pSmad $2 / 3$ at PCT by 1 D11, a TGF- $\beta$-neutralizing antibody, indicates active TGF- $\beta$ is high at the region of PCT, we cannot exclude other TGF- $\beta$ family ligands, e.g., activin.

Most importantly, deletion of Tgfb2r in Nestin lineage cells attenuated enthesis degeneration. The precise role of TGF- $\beta$ in the bone-tendon unit, however, appears to be complicated, perhaps with spatially and temporally dependent roles. Specifically, control of TGF- $\beta$ signaling has been shown to be both beneficial and detrimental, depending on the models utilized. Inhibition of the TGF- $\beta$ type 1 receptor demonstrated promising results for tendon repair 
after injuries (60). However, in a rat rotator cuff tendon-to-bone healing model, in which the enthesis was completely resected and the tendon was then reattached to the bone, inhibition of various TGF- $\beta$ isoforms did not lead to regenerative healing (64). In our study, we purposely preserved the Achilles tendon enthesis by partially transecting the Achilles tendon, leaving the native enthesis intact to specifically study the initial phase of enthesopathy, which may account for our different results. Inflammation may have a role in enthesopathy as well. We did not comprehensively explore inflammatory cytokines, as TNF- $\alpha$ and IL- $1 \beta$ in the PCT were unaffected in our models. There are possible limitations of the models, as neither is a true overuse model and these may not completely represent all of the changes in human enthesopathy.

High levels of active TGF- $\beta$ and increased osteoclast bone resorption were detected in PCT bone marrow at the onset of enthesopathy in animal models. Osteoclast bone resorption is known to allow active TGF- $\beta$ to be released from the bone matrix $(31,36)$. Inhibiting bone resorption may thus prevent subsequent activation of TGF- $\beta$ from matrix. Inhibition of bone resorption utilizing bisphosphonates has been shown to reduce bone mineral loss at anterior cruciate ligament entheses after joint injury (65). However, TGF- $\beta$, as a crucial growth factor, may be activated by means other than osteoclast bone resorption and plays an important role in a number of processes: inflammation, cell migration, stimulating angiogenesis, epithelial mesenchymal transition (EMT), endothelial mesenchymal transition (EndoMT), and remodeling of the new ECM (66-69). Therefore, drugs targeting osteoclast resorption may not be as effective as directly targeting TGF- $\beta$ signaling and would need further preclinical studies to validate. Tissue-oriented therapy that specifically inhibits TGF- $\beta$ activity would be a possible approach for treating enthesopathy. Indeed, we found that systemic administration of the TGF- $\beta$ antibody attenuated the initiation and progression of enthesopathy in various mouse models. There are several candidate drugs currently in use clinically or in clinical trials to consider for future preclinical or clinical trials of enthesopathy (70). Losartan, an angiotensin receptor blocker that is known to suppress the TGF- $\beta$-signaling cascade, is used clinically in Loeys-Dietz syndrome, Marfan syndrome, and CED, with encouraging outcomes $(71,72)$. There are also multiple drugs that more specifically target the TGF- $\beta$-signaling pathway being explored in clinical trials, such as fresolimumab and galunisertib (73-77). The most recent results utilizing a TGF- $\beta$ monoclonal antibody were in a phase 2 trial for systemic sclerosis and suggest the TGF- $\beta$ antibody has a reasonable safety profile (76). However, due to the complex and understudied etiology of human enthesopathy, the correlation between elevated TGF- $\beta$ signaling and human enthesopathy disease still remains unresolved. There are also concerns regarding blockade of TGF- $\beta$ signaling. For example, chronic, nonhealing wounds often show a loss of TGF- $\beta 1$ signaling $(78,79)$. Therefore, a delay in wound healing could be of potential concern upon TGF- $\beta$ agonist treatment. Further translational studies could be done efficiently for enthesopathy and are needed to explore the positive and negative effects more thoroughly.

Given the increasing incidence of tendon injuries (80), identifying and initiating treatment before tendon rupture is paramount. Our study identified that excessive activation of bone TGF- $\beta$ after partial injury to the Achilles tendon contributes to the pathogenesis of enthesopathy. Targeting the TGF- $\beta$-signaling pathway could help the fibrocartilaginous entheses maintain its mechanical force transmission function after tendon injuries.

\section{Methods}

Mice. We purchased C57BL/6J (WT) mice from the Jackson Laboratory. For the SMTS mouse model, we semitransected the Achilles tendon to generate a destabilized enthesopathy animal model adjusted as in a previously described procedure (63). Briefly, 3-month-old male mice were anesthetized by ketamine and xylazine followed by semitransection of Achilles tendon in the middle of the tendon body to induce abnormal mechanical loading-associated enthesopathy on the left calcaneus. Sham operations were done on independent mice. For dynamic histomorphometry analysis, we subcutaneously injected $0.1 \%$ calcein (MilliporeSigma, $10 \mathrm{mg} / \mathrm{kg}$ ) in PBS into the mice 10 and 3 days before euthanization. For the dosage-screening experiments, 3-month-old sham-operated and SMTS mice were assigned into 14 groups with 6 mice per group. Three days after surgery, we injected different doses $(1,3,5,10 \mathrm{mg} / \mathrm{kg})$ of TGF- $\beta$-neutralizing antibody (1D11) or the equivalent volume of vehicle antibody (13C4) intraperitoneally 3 times a week, once a week, and once a month for 30 days. All mice were euthanized 2 months after surgery ( $n=10$ per group).

For the DI model, TempAssure $0.5 \mathrm{ml}$ PCR tubes (USA Scientific, 1405-8100) were used. The bottoms ( $15 \mathrm{~mm}$ from the bottom) and the caps were removed from the tubes. Two holes were drilled $4 \mathrm{~mm}$ from the top of the tubes on opposite sides (180 degrees apart). The left ankle of a C57BL/6J (WT) mouse was inserted into the top of the tube in a dorsiflexion position. An iron wire $2 \mathrm{~cm}$ in length was utilized to secure the device in place, inserted in the 2 holes and above the ventral part of the ankle. When this device was applied, the calf muscles of the mice were tight. Therefore, the dorsiflexion of the ankle joint was restricted and the foot was bent into a talipes calcaneus position. The devices were applied to mice for 12 hours every day for 4 or 8 weeks as noted in Results. The mice were allowed to move freely in the cages. At the end of each experimental time point, the devices were discarded and mice were sacrificed. Nonimmobilized littermates were used as controls.

CED mice were generated in our laboratory as previously described. In these mice, the CED-derived TGF- $\beta 1$ mutation (H222D) is specifically expressed by osteoblastic cells driven by a 2.3-kb type I collagen promoter ( $n=10$ per group) (31). We purchased C57BL/6-Tg(Nes-cre/ERT2)KEisc/J (Nestin-cre/ERT2) mice and B6.129X1-Gt(ROSA)26Sor ${ }^{\text {tm1(EYFP)Cos/J }}$ (R26R-EYFP) mice from the Jackson Laboratory. Mice with floxed Tgfbr2 (Tgfbr $\left.2^{f / f f}\right)(\mathrm{C} 57 \mathrm{BL} / 6$ background) were obtained from the laboratory of H.L. Moses (Vanderbilt University, Nashville, Tennessee, USA) (81). Nestin-CreERT2 mice were crossed with $T g f b r 2^{f / f l}$ or $R 26 R$-EYFP mice. The offspring were intercrossed to generate Nestin-CreERT2::Tgfbr $2^{f / f l}$ and NestinCreERT2::R26R-EYFP offspring, respectively. We performed sham or SMTS operations on 3-month-old male Nestin-CreERT2::Tgfbr $2^{f / f l}$ and Nestin-CreERT2::R26R-EYFP mice. One day after surgery, we treated mice with either $100 \mathrm{mg}$ per $\mathrm{kg}$ body weight of tamoxifen or vehicle once a week for 4 weeks and euthanized the mice 8 weeks after surgery ( $n=10$ per group). All animals were maintained in the Animal Facility of the Johns Hopkins University School of Medicine.

Specimen collection. Mice were euthanized and perfusion fixed with $10 \%$ buffered formalin via the left ventricle for 5 minutes. Then 
we dissected the ankles including Achilles tendons and fixed the specimens in $10 \%$ buffered formalin for 24 hours. For histological and immunohistological examination, the fixed specimens were then decalcified in 10\% EDTA (pH 7.0) for 3 to 4 days and embedded in paraffin, OCT compound (VWR, 25608-930), or medium containing 8\% gelatin (Sigma-Aldrich, G1890) and 2\% polyvinylpyrrolidone (SigmaAldrich, PVP40) at $-80^{\circ} \mathrm{C}$. The majority of analyses were in paraffinembedded specimens, while detection of Nestin and CD31 was more optimal in frozen specimens. For dynamic histomorphometry analysis, the fixed samples were dehydrated and embedded in methylmethacrylate without decalcification.

Histology and morphometric analysis. Blocks were sectioned at 4 $\mu \mathrm{m}$ or $80 \mu \mathrm{m}$ (for CD31 immunofluorescent staining) intervals using a Microm cryostat (for frozen blocks) or a paraffin microtome (for paraffin blocks). The sections being compared were taken at the same anatomical level to ensure that the following experiments were conducted on serial sections and stained. For frozen tissue sections, slides were embedded in OCT compound (for $4-\mu \mathrm{m}$-thick slide) or gelatin (for $80-\mu \mathrm{m}$ thick slide) for 10 minutes at $-20^{\circ} \mathrm{C}$. For paraffin sections, slides were dewaxed in xylene. Both dewaxed paraffin sections and frozen sections were heated to $99^{\circ} \mathrm{C}$ for 20 minutes in citrate buffer (10 mM, pH 6.0) for antigen retrieval, and rehydrated. After washing 3 times with PBS, tissue sections were incubated with primary antibodies diluted in blocking solution (10\% BSA in PBS) overnight at $4^{\circ} \mathrm{C}$ in a humidified chamber. Sections were washed 3 times with Tris-buffered saline and incubated with secondary antibodies in blocking solution for 1 hour at room temperature. All immunofluorescence micrographs were acquired using an Axiovert 200M microscopy system (Carl Zeiss MicroImaging). Images were captured using Velocity software, and quantifications were performed using Image J software (NIH).

Either $4-\mu \mathrm{m}$-thick or $80-\mu \mathrm{m}$-thick (for CD31 and Emcn immunofluorescent staining) sagittal-oriented sections of the Achilles tendon enthesis were processed for histology and morphometric analysis. We used $\times 10$ images to measure the area of the CF and UF after H\&E and SOFG staining. CF and UF were separated by the tidemark line. We measured the area from the interface between UF and Achilles tendon to tidemark as the thickness of UF and the area from tidemark to PCT as the thickness of CF.

TRAP staining was processed following the manufacturer's protocol (Sigma-Aldrich, 387A-1KT), followed by counterstaining with Methyl Green (Sigma-Aldrich, M884). Quantitative analysis of OCS/ BS was conducted in a blinded fashion with OsteoMeasureXP Software (OsteoMetrics Inc.).

Immunostaining was performed using a standard protocol. We incubated sections with primary antibodies to mouse nestin (Aves Labs Inc., 1:300, lot NES0407), leptin receptor (R\&D Systems, 1:200, AF497), osteocalcin (Abcam, ab93876, 1:200), osterix (Abcam, 1:600, ab22552), pSmad2/3 (Santa Cruz Biotechnology Inc., 1:50, sc-11769), CD31 (Abcam, 1:100, ab28364), Emcn (Santa Cruz Biotechnology Inc., V.7C7, 1:50), MMP13 (Abcam, 1:40, ab3208), collagen X (Abcam, 1:80, ab58632), TNF- $\alpha$ (Abcam, 1:100, ab6671), IL-1 (Abcam, 1:100, ab9722), and GFP (Abcam, ab290, 1:200) overnight at $4^{\circ} \mathrm{C}$. For immunohistochemical staining, we subsequently used a horseradish peroxidase-streptavidin detection system (Dako) to detect the immunoactivity, followed by counterstaining with hematoxylin (Sigma-Aldrich, H9627). For immunofluorescence staining, we continued to use secondary antibodies conjugated with red fluorescence, incubated the slides, avoiding light, at room temperature for 1 hour, and mounted the slides with ProLong Gold Mounting Reagent with DAPI (P36935, Life Technologies). Then we photographed sections to perform histomorphometric measurements on the entire area of Achilles tendon enthesis (Olympus, DP71). We conducted the quantitative analysis in a blinded fashion with OsteoMeasureXP Software (OsteoMetrics Inc.).

Calcein double labeling in undecalcified slices was observed under a fluorescence microscope. Dynamic parameters such as mineral apposition rate (MAR) and bone formation rate (BFR) were measured in 8 randomly selected visual fields.

TUNEL assays were performed with an In Situ Cell Death Detection Kit (S7110, EMD Millipore) according to the manufacturer's instructions. Briefly, middle Achilles tendon sections from DI models were deparaffinized, rehydrated, and incubated with $50 \mu \mathrm{l}$ of TUNEL reaction mixture. Images were acquired using an Olympus BX51 microscope and positive cells were quantified with OsteoMeasureXP Software (OsteoMetrics Inc.).

Serum TGF- $\beta 1$ and osteocalcin analysis. We determined the concentration of active TGF- $\beta 1$ in the mouse serum using the ELISA Development Kit (R\&D Systems, MB100B) under the manufacturer's instructions. For total TGF- $\beta 1$ measurement, samples were first treated with $1 \mathrm{~N} \mathrm{HCl}(1 \mu \mathrm{l} / 50 \mu \mathrm{l}$ supernatant) for 15 minutes at room temperature and were neutralized with an equal amount of $1 \mathrm{~N} \mathrm{NaOH}$ before analysis in ELISA. For active TGF- $\beta 1$ measurement, samples were analyzed in ELISA without acid treatment. We determined the concentration of osteocalcin in the mouse serum using the ELISA Development Kit (Alfa Aesar, J64239) according to the manufacturer's instructions.

$\mu C T$ analysis. L4 vertebral bodies and ankles with feet and Achilles tendons were dissected free of surrounding tissue from mice, then fixed overnight in 10\% formalin and analyzed by high-resolution $\mu \mathrm{CT}$ (Skyscan1172). The images were reconstructed and analyzed by NRecon v1.6 and CTAn v1.9, respectively. We analyzed parameters using 3D model visualization software, CTVol v2.0. The scanner was set at a voltage of $60 \mathrm{kV}$ and a resolution of $5.78 \mu \mathrm{m}$ per pixel. Images of PCT were used to perform 3D histomorphometric analyses. The region of interest was defined to cover the whole PCT compartment (from the posterior tip of the calcaneus to $1 \mathrm{~mm}$ anterior to the posterior tip of the calcaneus). We used a total of 8 consecutive images for 3D reconstruction. 3D structural parameters analyzed included BV/TV, Tb.Th, Tb.N, Tb.Sp, and Tb.Pf.

CT-based microangiography. Angiography of microphil-perfused bones was performed to image blood vessels in bone. Briefly, after the mice were euthanized, the vascular system was flushed with $0.9 \%$ normal saline solution containing heparin sodium $(100 \mathrm{U} / \mathrm{ml})$ through a needle inserted into the left ventricle, followed by fixation with $10 \%$ neutral buffered formalin. After washing 3 times using 0.9\% normal saline solution, radiopaque silicone rubber compound containing lead chromate (MICROFIL MV-122, Flow Tech) was injected. Specimens were stored at $4^{\circ} \mathrm{C}$ overnight for contrast agent polymerization. Ankles with feet were then dissected and soaked in $10 \%$ neutral buffered formalin for 4 days, followed by decalcification in a formic acid-based solution (Cal-Ex II) for 48 hours to facilitate image threshold of the vasculature from the surrounding tissues. A high-resolution $\mu \mathrm{CT}$ imaging system (Skyscan 1172) was employed to acquire images. The scanner was set at a voltage of $60 \mathrm{kV}$ and a resolution of $5.78 \mu \mathrm{m}$ per pixel.

Statistics. All statistical analyses were carried out using SPSS 15 software. Data are presented as mean \pm SEM. We performed compar- 
isons using 2-tailed Student's $t$ test (for comparison of WT and CED mice) or 1-way ANOVA, followed by Tukey's post-hoc test (for all the other comparisons) to determine significance between groups. The level of significance was set at $P<0.05$.

Study approval. The experimental protocols were reviewed and approved by the Institutional Animal Care and Use Committee of Johns Hopkins University.

\section{Author contributions}

XW conceived the ideas for experimental designs, conducted the majority of the experiments, analyzed data, and prepared the manuscript. LX conducted some of the surgery and performed statistical analysis. JC and GZ provided critical suggestions for the project and helped compose the manuscript. FL, PY, and MG provided $\mu \mathrm{CT}$ analysis. RD, YW, and XJ performed DI model analysis. CF and MW provided suggestions for the project. XC developed the concept, supervised the project, and conceived the experiments.

\section{Acknowledgments}

This work was supported in part by NIH/National Institute of Arthritis and Musculoskeletal and Skin Diseases grants AR071432 and AR063943 (to XC).

Address correspondence to: $\mathrm{Xu}$ Cao, 720 Rutland Avenue, Ross 231, School of Medicine, Johns Hopkins University, Baltimore, Maryland, USA. Phone: 410.502.6440; Email: xcao11@jhmi.edu.
1. Genin GM, et al. Functional grading of mineral and collagen in the attachment of tendon to bone. Biophys J. 2009;97(4):976-985.

2. Benjamin M, Toumi H, Ralphs JR, Bydder G, Best TM, Milz S. Where tendons and ligaments meet bone: attachment sites ('entheses') in relation to exercise and/or mechanical load. J Anat. 2006;208(4):471-490.

3. Ristolainen L, Kettunen JA, Kujala UM, Heinonen A. Sport injuries as the main cause of sport career termination among Finnish top-level athletes. Eur J Sport Sci. 2012;12(3):274-282.

4. Järvinen TA, Kannus P, Maffulli N, Khan KM. Achilles tendon disorders: etiology and epidemiology. Foot Ankle Clin. 2005;10(2):255-266.

5. Józsa L, Kannus P. Histopathological findings in spontaneous tendon ruptures. Scand JMed Sci Sports. 1997;7(2):113-118.

6. Lee KS. Musculoskeletal sonography of the tendon. JUltrasound Med. 2012;31(12):1879-1884.

7. Benjamin M, McGonagle D. The anatomical basis for disease localisation in seronegative spondyloarthropathy at entheses and related sites. JAnat. 2001;199(Pt 5):503-526.

8. Hibino N, Hamada Y, Sairyo K, Yukata K, Sano $\mathrm{T}$, Yasui N. Callus formation during healing of the repaired tendon-bone junction. A rat experimental model. J Bone Joint Surg Br. 2007;89(11):1539-1544.

9. Raspanti M, Strocchi R, De Pasquale V, Martini D, Montanari C, Ruggeri A. Structure and ultrastructure of the bone/ligament junction. Ital J Anat Embryol. 1996;101(2):97-105.

10. François RJ. Ligament insertions into the human lumbar vertebral body. Acta Anat (Basel). 1975;91(3):467-480.

11. McGonagle D, et al. Histological assessment of the early enthesitis lesion in spondyloarthropathy. Ann Rheum Dis. 2002;61(6):534-537.

12. Mariotti V, Facchini F, Belcastro MG. Enthesopathies--proposal of a standardized scoring method and applications. Coll Antropol. 2004;28(1):145-159.

13. Havelková P, Villotte S. Enthesopathies: test of reproducibility of the new scoring system based on current medical data. Slovenská antropológia. 2007;10(1):51-57.

14. Kumagai J, Sarkar K, Uhthoff HK, Okawara Y, Ooshima A. Immunohistochemical distribution of type I, II and III collagens in the rabbit supra- spinatus tendon insertion. J Anat. 1994; 185(Pt 2):279-284.

15. Sagarriga Visconti C, Kavalkovich K, Wu J, Niyibizi C. Biochemical analysis of collagens at the ligament-bone interface reveals presence of cartilage-specific collagens. Arch Biochem Biophys. 1996;328(1):135-142.

16. Fukuta S, Oyama M, Kavalkovich K, Fu FH, Niyibizi C. Identification of types II, IX and X collagens at the insertion site of the bovine achilles tendon. Matrix Biol. 1998;17(1):65-73.

17. Waggett AD, Ralphs JR, Kwan AP, Woodnutt D, Benjamin M. Characterization of collagens and proteoglycans at the insertion of the human Achilles tendon. Matrix Biol. 1998;16(8):457-470.

18. Thomopoulos S, Williams GR, Gimbel JA, Favata M, Soslowsky LJ. Variation of biomechanical, structural, and compositional properties along the tendon to bone insertion site. JOrthop Res. 2003;21(3):413-419.

19. Zhang YG, Sun ZM, Liu JT, Wang SJ, Ren FL, Guo X. Features of intervertebral disc degeneration in rat's aging process. JZhejiang Univ Sci B. 2009;10(7):522-527.

20. Roberts S, Caterson B, Menage J, Evans EH, Jaffray DC, Eisenstein SM. Matrix metalloproteinases and aggrecanase: their role in disorders of the human intervertebral disc. Spine. 2000;25(23):3005-3013.

21. Le Maitre CL, Freemont AJ, Hoyland JA. Localization of degradative enzymes and their inhibitors in the degenerate human intervertebral disc. J Pathol. 2004;204(1):47-54.

22. Bastow ER, Byers S, Golub SB, Clarkin CE, Pitsillides AA, Fosang AJ. Hyaluronan synthesis and degradation in cartilage and bone. Cell Mol Life Sci. 2008;65(3):395-413.

23. Milz S, et al. Molecular composition and pathology of entheses on the medial and lateral epicondyles of the humerus: a structural basis for epicondylitis. Ann Rheum Dis. 2004;63(9):1015-1021.

24. Resnick D, Feingold ML, Curd J, Niwayama G, Goergen TG. Calcaneal abnormalities in articular disorders. Rheumatoid arthritis, ankylosing spondylitis, psoriatic arthritis, and Reiter syndrome. Radiology. 1977;125(2):355-366.

25. Bassiouni M. Incidence of calcaneal spurs in osteo-arthrosis and rheumatoid arthritis, and in control patients. Ann Rheum Dis. 1965;24(5):490-493.
26. Rubin G, Witten M. Plantar calcaneal spurs. Am J Orthop. 1963;5:38-41.

27. Zhang F, et al. Transforming growth factor-beta promotes recruitment of bone marrow cells and bone marrow-derived mesenchymal stem cells through stimulation of MCP-1 production in vascular smooth muscle cells. J Biol Chem. 2009;284(26):17564-17574.

28. Wan $\mathrm{M}$, et al. Injury-activated transforming growth factor $\beta$ controls mobilization of mesenchymal stem cells for tissue remodeling. Stem Cells. 2012;30(11):2498-2511.

29. Crane JL, Cao X. Bone marrow mesenchymal stem cells and TGF- $\beta$ signaling in bone remodeling. J Clin Invest. 2014;124(2):466-472.

30. Zhen G, Cao X. Targeting TGF $\beta$ signaling in subchondral bone and articular cartilage homeostasis. Trends Pharmacol Sci. 2014;35(5):227-236.

31. Tang Y, et al. TGF-beta1-induced migration of bone mesenchymal stem cells couples bone resorption with formation. Nat Med. 2009;15(7):757-765.

32. Gao P, et al. Functional effects of TGF- $\beta 1$ on mesenchymal stem cell mobilization in cockroach allergen-induced asthma. Jimmunol. 2014;192(10):4560-4570.

33. Yang X, Chen L, Xu X, Li C, Huang C, Deng CX. TGF-beta/Smad3 signals repress chondrocyte hypertrophic differentiation and are required for maintaining articular cartilage. J Cell Biol. 2001;153(1):35-46.

34. Shinojima N, et al. TGF- $\beta$ mediates homing of bone marrow-derived human mesenchymal stem cells to glioma stem cells. Cancer Res. 2013;73(7):2333-2344.

35. Grafe I, et al. Excessive transforming growth factor- $\beta$ signaling is a common mechanism in osteogenesis imperfecta. Nat Med. 2014;20(6):670-675.

36. Zhen $G$, et al. Inhibition of TGF- $\beta$ signaling in mesenchymal stem cells of subchondral bone attenuates osteoarthritis. Nat Med. 2013;19(6):704-712.

37. Braun J, et al. Use of immunohistologic and in situ hybridization techniques in the examination of sacroiliac joint biopsy specimens from patients with ankylosing spondylitis. Arthritis Rheum. 1995;38(4):499-505

38. Claudepierre $\mathrm{P}$, et al. A relationship between TGF-beta 1 or IL-6 plasma levels and clinical features of spondyloarthropathies. Br J Rheumatol. 
1997;36(3):400-401.

39. Maffulli N. Rupture of the Achilles tendon. JBone Joint Surg Am. 1999;81(7):1019-1036.

40. Hahn M, Vogel M, Pompesius-Kempa M, Delling G. Trabecular bone pattern factor--a new parameter for simple quantification of bone microarchitecture. Bone. 1992;13(4):327-330.

41. Sarahrudi K, et al. Elevated transforming growth factor-beta 1 (TGF- $\beta 1$ ) levels in human fracture healing. Injury. 2011;42(8):833-837.

42. Quinlan JF, Watson RW, Kelly G, Kelly PM, O'Byrne JM, Fitzpatrick JM. Transforming growth factor-beta (TGF-beta) in acute injuries of the spinal cord. J Bone Joint Surg Br. 2006;88(3):406-410.

43. Morganti-Kossmann MC, et al. TGF-beta is elevated in the CSF of patients with severe traumatic brain injuries and parallels blood-brain barrier function. J Neurotrauma. 1999;16(7):617-628.

44. Varedi M, Jeschke MG, Englander EW, Herndon DN, Barrow RE. Serum TGF-beta in thermally injured rats. Shock. 2001;16(5):380-382.

45. Castagnola P, Moro G, Descalzi-Cancedda F, Cancedda R. Type $\mathrm{X}$ collagen synthesis during in vitro development of chick embryo tibial chondrocytes. J Cell Biol. 1986;102(6):2310-2317.

46. Chen QA, Gibney E, Fitch JM, Linsenmayer C, Schmid TM, Linsenmayer TF. Long-range movement and fibril association of type $\mathrm{X}$ collagen within embryonic cartilage matrix. Proc Natl Acad Sci U S A. 1990;87(20):8046-8050.

47. Méndez-Ferrer S, et al. Mesenchymal and haematopoietic stem cells form a unique bone marrow niche. Nature. 2010;466(7308):829-834.

48. Ono N, Ono W, Mizoguchi T, Nagasawa T, Frenette PS, Kronenberg HM. Vasculature-associated cells expressing nestin in developing bones encompass early cells in the osteoblast and endothelial lineage. Dev Cell. 2014;29(3):330-339.

49. Itkin $\mathrm{T}$, et al. Distinct bone marrow blood vessels differentially regulate haematopoiesis. Nature. 2016;532(7599):323-328.

50. Zhou BO, Yue R, Murphy MM, Peyer JG, Morrison SJ. Leptin-receptor-expressing mesenchymal stromal cells represent the main source of bone formed by adult bone marrow. Cell Stem Cell. 2014;15(2):154-168

51. Kusumbe AP, Ramasamy SK, Adams RH. Coupling of angiogenesis and osteogenesis by a specific vessel subtype in bone. Nature. 2014;507(7492):323-328.

52. Astrom M, Rausing A. Chronic Achilles tendinopathy. A survey of surgical and histopathologic findings. Clin Orthop Relat Res. 1995;(316):151-164.

53. Myerson MS, McGarvey W. Disorders of the
Achilles tendon insertion and Achilles tendinitis. Instr Course Lect. 1999;48:211-218.

54. Blaney Davidson EN, van der Kraan PM, van den Berg WB. TGF-beta and osteoarthritis. Osteoarthr Cartil. 2007;15(6):597-604.

55 . Shi M, et al. Latent TGF- $\beta$ structure and activation. Nature. 2011;474(7351):343-349.

56. Zhang M, Wang M, Tan X, Li TF, Zhang YE, Chen D. Smad3 prevents beta-catenin degradation and facilitates beta-catenin nuclear translocation in chondrocytes. J Biol Chem. 2010;285(12):8703-8710.

57. Li TF, et al. Smad3-deficient chondrocytes have enhanced BMP signaling and accelerated differentiation. J Bone Miner Res. 2006;21(1):4-16.

58. Vogel KG, Koob TJ. Structural specialization in tendons under compression. Int Rev Cytol. 1989;115:267-293.

59. Robbins JR, Evanko SP, Vogel KG. Mechanical loading and TGF-beta regulate proteoglycan synthesis in tendon. Arch Biochem Biophys. 1997;342(2):203-211.

60. Maeda T, et al. Conversion of mechanical force into TGF- $\beta$-mediated biochemical signals. Curr Biol. 2011;21(11):933-941.

61. Chen R, et al. Attenuation of the progression of articular cartilage degeneration by inhibition of TGF- $\beta 1$ signaling in a mouse model of osteoarthritis. Am J Pathol. 2015;185(11):2875-2885.

62. Waning DL, et al. Excess TGF- $\beta$ mediates muscle weakness associated with bone metastases in mice. Nat Med. 2015;21(11):1262-1271.

63. McClure J. The effect of diphosphonates on heterotopic ossification in regenerating Achilles tendon of the mouse. J Pathol.1983;139(4):419-430.

64. Kim HM, Galatz LM, Das R, Havlioglu N, Rothermich SY, Thomopoulos S. The role of transforming growth factor beta isoforms in tendon-to-bone healing. Connect Tissue Res. 2011;52(2):87-98.

65. Doschak MR, et al. Bisphosphonates reduce bone mineral loss at ligament entheses after joint injury. Osteoarthr Cartil. 2005;13(9):790-797.

66. Roberts AB, et al. Transforming growth factor type beta: rapid induction of fibrosis and angiogenesis in vivo and stimulation of collagen formation in vitro. Proc Natl Acad Sci U S A 1986;83(12):4167-4171.

67. Nall AV, et al. Transforming growth factor beta 1 improves wound healing and random flap survival in normal and irradiated rats. Arch Otolaryngol Head Neck Surg. 1996;122(2):171-177.

68. Piera-Velazquez S, Li Z, Jimenez SA. Role of endothelial-mesenchymal transition (EndoMT) in the pathogenesis of fibrotic disorders. Am J
Pathol. 2011;179(3):1074-1080.

69. Xu J, Lamouille S, Derynck R. TGF-beta-induced epithelial to mesenchymal transition. Cell Res. 2009;19(2):156-172.

70. Matt P, Habashi J, Carrel T, Cameron DE, Van Eyk JE, Dietz HC. Recent advances in understanding Marfan syndrome: should we now treat surgical patients with losartan? J Thorac Cardiovasc Surg. 2008;135(2):389-394.

71. MacCarrick G, et al. Loeys-Dietz syndrome: a primer for diagnosis and management. Genet Med. 2014;16(8):576-587.

72. Ayyavoo A, Derraik JG, Cutfield WS, Hofman PL. Elimination of pain and improvement of exercise capacity in Camurati-Engelmann disease with losartan. JClin Endocrinol Metab. 2014;99(11):3978-3982

73. Morris JC, et al. Phase I study of GC1008 (fresolimumab): a human anti-transforming growth factorbeta (TGF $\beta$ ) monoclonal antibody in patients with advanced malignant melanoma or renal cell carcinoma. PLoS One. 2014;9(3):e90353.

74. Trachtman H, et al. A phase 1, single-dose study of fresolimumab, an anti-TGF- $\beta$ antibody, in treatment-resistant primary focal segmental glomerulosclerosis. Kidney Int. 2011;79(11):1236-1243.

75. Lacouture ME, et al. Cutaneous keratoacanthomas/squamous cell carcinomas associated with neutralization of transforming growth factor $\beta$ by the monoclonal antibody fresolimumab (GC1008). Cancer Immunol Immunother. 2015;64(4):437-446.

76. Rice LM, et al. Fresolimumab treatment decreases biomarkers and improves clinical symptoms in systemic sclerosis patients. JClin Invest. 2015;125(7):2795-2807.

77. Herbertz S, et al. Clinical development of galunisertib (LY2157299 monohydrate), a small molecule inhibitor of transforming growth factorbeta signaling pathway. Drug Des Devel Ther. 2015;9:4479-4499.

78. Pastar I, et al. Attenuation of the transforming growth factor beta-signaling pathway in chronic venous ulcers. Mol Med. 2010;16(3-4):92-101.

79. Kim BC, et al. Fibroblasts from chronic wounds show altered TGF-beta-signaling and decreased TGF-beta Type II receptor expression. JCell Physiol. 2003;195(3):331-336

80. Nyyssönen T, Lüthje P, Kröger H. The increasing incidence and difference in sex distribution of Achilles tendon rupture in Finland in 1987-1999. Scand J Surg. 2008;97(3):272-275.

81. Chytil A, Magnuson MA, Wright CV, Moses HL. Conditional inactivation of the TGF-beta type II receptor using Cre:Lox. Genesis. 2002;32(2):73-75. 